TRANSACTIONS OF THE

AMERICAN MATHEMATICAL SOCIETY

Volume 363, Number 4, April 2011, Pages 1765-1788

S 0002-9947(2010)05164-5

Article electronically published on November 8, 2010

\title{
REAL FORMS OF COMPLEX SURFACES OF CONSTANT MEAN CURVATURE
}

\author{
SHIMPEI KOBAYASHI
}

\begin{abstract}
It is known that complex constant mean curvature (CMC for short) immersions in $\mathbb{C}^{3}$ are natural complexifications of CMC-immersions in $\mathbb{R}^{3}$. In this paper, conversely we consider real form surfaces of a complex CMC-immersion, which are defined from real forms of the twisted $\mathfrak{s l}(2, \mathbb{C})$ loop algebra $\Lambda \mathfrak{s l}(2, \mathbb{C})_{\sigma}$, and classify all such surfaces according to the classification of real forms of $\Lambda \mathfrak{s l}(2, \mathbb{C})_{\sigma}$. There are seven classes of surfaces, which are called integrable surfaces, and all integrable surfaces will be characterized by the (Lorentz) harmonicities of their Gauss maps into the symmetric spaces $S^{2}$, $H^{2}, S^{1,1}$ or the 4 -symmetric space $S L(2, \mathbb{C}) / U(1)$. We also give a unification to all integrable surfaces via the generalized Weierstrass type representation.
\end{abstract}

\section{INTRODUCTION}

The goal of this paper is to give a unified theory for integrable surfaces using the real forms of complex extended framings of complex CMC-immersions and the generalized Weierstrass type representation for complex CMC-immersions.

It is classically known that CMC surfaces with nonzero mean curvature (or equivalently constant positive Gaussian curvature (CPC for short) surfaces as parallel surfaces) and constant negative Gaussian curvature (CNC for short) surfaces in $\mathbb{R}^{3}$ are characterized by the transformations of real (or complex) tangential line congruences between surfaces with special properties, which are commonly called "(Bianchi) Bäcklund transformations". In modern terminology, such classes of surfaces are characterized by the (Lorentz) harmonicities of their Gauss maps, and they are equivalent to the existence of families of flat connections on $\mathcal{M} \times S O(3)$, where $\mathcal{M}$ is $\mathbb{C}$ for $\mathrm{CMC}$-immersions or $\mathbb{R}^{1,1}$ for CNC-immersions; see [1] and [19]. Spacelike or timelike constant positive or negative Gaussian curvature surfaces in $\mathbb{R}^{1,2}$ are less known, however, they are also characterized by the (Lorentz) harmonicities of their Gauss maps, or equivalently, the existence of families of flat connections on $\mathcal{M} \times S O(2,1)$, where $\mathcal{M}$ is $\mathbb{C}$ for spacelike $\mathrm{CNC}$-immersions (or equivalently spacelike CMC-immersions) and timelike CNC-immersions, or $\mathbb{R}^{1,1}$ for spacelike CPC-immersions and timelike CPC-immersions (or equivalently timelike CMCimmersions); see 8 and 20 .

On the one hand, to classify all CMC-cylinders in $\mathbb{R}^{3}$, in $[9$ we gave a natural complexification of the extended framing, a moving frame with spectral parameter and an element in the $S U(2)$ loop group, of a CMC-immersion, which is called the

Received by the editors June 3, 2008.

2000 Mathematics Subject Classification. Primary 53A10.

(C)2010 American Mathematical Society Reverts to public domain 28 years from publication 
complex extended framing. Moreover in [10, we introduced holomorphic immersions in $\mathbb{C}^{3}$ associated with the complex extended framings and a natural definition of the complex mean curvature for a holomorphic immersion. Then a holomorphic immersion with complex constant mean curvature $H \in \mathbb{C}$ is naturally called the complex CMC-immersion. Similar to the real case, a holomorphic immersion with complex constant Gauss curvature $K \in \mathbb{C}^{*}$ (CGC for short) is obtained as the parallel immersion of a complex CMC-immersion with nonzero complex constant mean curvature $H \in \mathbb{C}^{*}$.

In this paper, we shall interpret those complex CGC-immersions (or equivalently CMC-immersions by the parallel immersions) as complexifications for the surfaces discussed above. These real surfaces are then obtained by the real form surfaces of a complex CGC-immersion, which are defined from the real forms of the MaurerCartan form of the complex extended framing of a complex CMC-immersion. It is known that the twice central extensions of a loop algebra $\Lambda \mathfrak{s l}(2, \mathbb{C})_{\sigma}$ is a twisted affine Kac-Moody Lie algebra of $A_{1}^{(1)}$ type. The classification of the real forms of affine Kac-Moody Lie algebras was given in [3] and [4. It follows that the classification of real forms of $\Lambda \mathfrak{s l}(2, \mathbb{C})_{\sigma}$ is also given. In particular, the real forms of $\Lambda \mathfrak{s l}(2, \mathbb{C})_{\sigma}$ consist of seven classes: three classes are called the almost split and the other four classes are called the almost compact, according to the types of the semi-linear involutions of the real forms. Thus there are seven classes of surfaces, which are called integrable surfaces, according to the classification of the real forms of $\Lambda \mathfrak{s l}(2, \mathbb{C})_{\sigma}$. Spacelike or timelike CMC or CGC surfaces in $\mathbb{R}^{3}$ or $\mathbb{R}^{1,2}$ form the six classes of integrable surfaces, and CMC surfaces with mean curvature $|H|<1$ in $H^{3}$ form the last class of integrable surfaces (Theorem 3.3 and Corollary [3.4). Moreover, all integrable surfaces are characterized by (Lorentz) harmonicities of their Gauss maps, which are maps into symmetric spaces $S^{2}, H^{2}, S^{1,1}$ and the 4 -symmetric space $S L(2, \mathbb{C}) / U(1)$, respectively (Theorem 3.6).

The generalized Weierstrass type representation for complex CMC-immersions is a procedure to construct complex CMC-immersions in $\mathbb{C}^{3}$ (see Section 4.1 for more details): 1. Define pairs of holomorphic potentials, which are pairs of holomorphic 1-forms $\check{\eta}=(\eta, \tau)$ with $\eta=\sum_{j \geq-1}^{\infty} \eta_{j} \lambda^{j}$ and $\tau=\sum_{-\infty}^{j \leq 1} \tau_{j} \lambda^{j}$. Here $\lambda$ is the complex parameter, the so-called "spectral parameter", $\eta_{j}$ and $\tau_{j}$ are diagonal (resp. off-diagonal) holomorphic 1-forms depending only on one complex variable if $j$ is even (resp. $j$ is odd). 2. Solve the pair of ODEs $d(C, L)=(C, L) \check{\eta}$ with some initial condition $\left(C\left(z_{*}\right), L\left(w_{*}\right)\right)$, and perform the generalized Iwasawa decomposition (Theorem A.2) for $(C, L)$, giving $(C, L)=(F, F)\left(V_{+}, V_{-}\right)$. It is known that $F \cdot l$ is the complex extended framing of some complex CMC-immersion (Theorem 4.1), where $l$ is some $\lambda$-independent diagonal matrix. 3. Form a complex CMCimmersion by the Sym formula $\Psi$ via the complex extended framing $F \cdot l$ (Theorem 2.6).

Since each class of integrable surfaces is defined by a real form of $\Lambda \mathfrak{s l}(2, \mathbb{C})_{\sigma}$, there exists a unique semi-linear involution $\rho$ corresponding to each class of integrable surfaces. Then these semi-linear involutions naturally define the pairs of semilinear involutions on pairs of holomorphic potentials $\check{\eta}=(\eta, \tau)$. It follows that the generalized Weierstrass type representation for each class of integrable surfaces can be formulated by the above construction with a pair of holomorphic potentials which is invariant under a pair of semi-linear involutions (Theorem 4.2). In this way we give a unified theory for all integrable surfaces. 
More precisely, in Section 2, we give a brief review of the basic results for complex CMC and CGC-immersions. In Section 2.1, holomorphic null immersions and the basic facts for holomorphic null immersions are considered. In Section 2.2 the basic facts and results for complex CMC-immersions are given. Analogously to the complex CMC-immersions, the definition and the basic facts for complex CGCimmersions are given (Theorem 2.6). In Section 2.3, the classification of real forms of $\Lambda \mathfrak{s l}(2, \mathbb{C})_{\sigma}$ is given (Theorem 2.10 and Theorem 2.11).

In Section 3, we give a classification of integrable surfaces. In Section 3.1, it is shown that all integrable surfaces are obtained from real forms of $\Lambda \mathfrak{s l}(2, \mathbb{C})_{\sigma}$ (Theorem 3.3). In Section 3.2, the Gauss maps of integrable surfaces are characterized using the Gauss map of a complex CMC-immersion and the real forms of the complex extended framing (Theorem 3.6).

In Section 4 we give a construction of all integrable surfaces via the generalized Weierstrass type representation. In Section 4.1 the pairs of semi-linear involutions, which are determined from classes of integrable surfaces, are considered. Then a construction of all integrable surfaces is discussed via the pairs of holomorphic potentials which are invariant under the pairs of semi-linear involutions (Theorem 4.2).

In Appendix A, we give the basic results for affine Kac-Moody Lie algebras and the loop algebras. In Section A.1 Kac-Moody Lie algebras are considered. In Section A.2, loop groups and a realization of affine Kac-Moody Lie algebras via the twice central extensions of loop algebras are discussed (Theorem A.1). In Section A.3, double loop groups are defined and the Iwasawa decomposition theorem for the double loop groups are given (Theorem A.2).

\section{Preliminaries}

In this preliminary section, we give a brief review of the basic results for holomorphic null immersions, complex CMC-immersions and complex CGC-immersions. We also give a brief review of the basic facts about loop algebras and their real forms.

Throughout this paper, $\mathbb{C}^{3}$ is identified with $\mathfrak{s l}(2, \mathbb{C})$ as follows:

$$
(a, b, c)^{t} \in \mathbb{C}^{3} \leftrightarrow-\frac{i a}{2} \sigma_{1}-\frac{i b}{2} \sigma_{2}-\frac{i c}{2} \sigma_{3} \in \mathfrak{s l}(2, \mathbb{C}),
$$

where $\sigma_{j}(j=1,2,3)$ are Pauli matrices as follows:

$$
\sigma_{1}=\left(\begin{array}{cc}
0 & 1 \\
1 & 0
\end{array}\right), \quad \sigma_{2}=\left(\begin{array}{cc}
0 & -i \\
i & 0
\end{array}\right) \text { and } \sigma_{3}=\left(\begin{array}{cc}
1 & 0 \\
0 & -1
\end{array}\right) .
$$

2.1. Holomorphic null immersions in $\mathbb{C}^{3}$. In this subsection, we show the basic results for holomorphic immersions $\Psi$ from $\mathfrak{D}^{2} \subset \mathbb{C}^{2}$ into $\mathbb{C}^{3}$. We give natural definitions of complex mean curvature (Definition 1) and complex Gauss curvature (Definition 2) for a holomorphic immersion analogous to the mean curvature and the Gauss curvature of a surface in $\mathbb{R}^{3}$. We refer to [10] for more details.

Let $\mathcal{M}$ be a simply connected 2-dimensional Stein manifold, and let $\Psi: \mathcal{M} \rightarrow$ $\mathfrak{s t}(2, \mathbb{C})$ be a holomorphic immersion, i.e., the complex rank of $d \Psi$ is two. We consider the following bilinear form on $\mathfrak{s l}(2, \mathbb{C}) \cong \mathbb{C}^{3}$ :

$$
\langle a, b\rangle=-2 \operatorname{Tr} a b,
$$


where $a, b \in \mathfrak{s l}(2, \mathbb{C})$. We note that the bilinear form (2.1.1) is a $\mathbb{C}$-bilinear form on $\mathbb{C}^{3}$ by the identification (2.0.1). Then it is known that, for a neighborhood $\widetilde{\mathcal{M}}_{p} \subset \mathcal{M}$ around each point $p \in \mathcal{M}$, the bilinear form (2.1.1) induces a holomorphic Riemannian metric on $\widetilde{\mathcal{M}}_{p}$, i.e., a holomorphic covariant symmetric 2-tensor $g$, see [18 and 10. From [10, it is also known that there exist special coordinates $(z, w) \in \mathfrak{D}^{2} \subset \mathbb{C}^{2}$ such that a holomorphic Riemannian metric $g$ can be written as follows:

$$
g=e^{u(z, w)} d z d w,
$$

where $u(z, w): \mathfrak{D}^{2} \rightarrow \mathbb{C}$ is some holomorphic function. The special coordinates defined above are called null coordinates. From now on, we always assume a holomorphic immersion $\Psi: \mathcal{M} \rightarrow \mathfrak{s l}(2, \mathbb{C})$ has null coordinates. A holomorphic immersion with null coordinates is also called the holomorphic null immersion.

Remark 2.1. The assumption "Stein" is used for the existence of the form of a holomorphic Riemannian metric $g=e^{u(z, w)} d z d w$ defined in (2.1.2) and the existence of a well-defined pair of holomorphic potentials on $\mathcal{M}$ for the generalized Weierstrass type representation in Section 4 see [10] for more details.

We now define a vector $N \in \mathfrak{s l}(2, \mathbb{C})$ as follows:

$$
N:=2 i e^{-u}\left[\Psi_{w}, \Psi_{z}\right],
$$

where the subscripts $z$ and $w$ denote the partial derivatives with respect to $z$ and $w$, respectively. It is easy to verify that $\left\langle\Psi_{z}, N\right\rangle=\left\langle\Psi_{w}, N\right\rangle=0$ and the $\langle N, N\rangle=1$. Thus $N$ is a transversal vector to $d \Psi$. Therefore it is natural to call $N$ the complex Gauss map of $\Psi$.

From [10, we quote the following theorem:

Theorem 2.2 (10]). Let $\Psi: \mathcal{M} \rightarrow \mathbb{C}^{3}(\cong \mathfrak{s l}(2, \mathbb{C}))$ be a holomorphic null immersion. Then there exists a $S L(2, \mathbb{C})$ matrix $F$ such that the following equations hold:

$$
\begin{aligned}
& F_{z}=F U, \\
& F_{w}=F V,
\end{aligned}
$$

where

$$
\left\{\begin{array}{c}
U=\left(\begin{array}{cc}
\frac{1}{4} u_{z} & -\frac{1}{2} H e^{u / 2} \\
Q e^{-u / 2} & -\frac{1}{4} u_{z}
\end{array}\right), \\
V=\left(\begin{array}{cc}
-\frac{1}{4} u_{w} & -R e^{-u / 2} \\
\frac{1}{2} H e^{u / 2} & \frac{1}{4} u_{w}
\end{array}\right),
\end{array}\right.
$$

with $Q:=\left\langle\Psi_{z z}, N\right\rangle, R:=\left\langle\Psi_{w w}, N\right\rangle$ and $H:=2 e^{-u}\left\langle\Psi_{z w}, N\right\rangle$.

We call $F: \mathcal{M} \rightarrow S L(2, \mathbb{C})$ the moving frame of $\Psi$. Then the compatibility condition for the equations in (2.1.4) is

$$
U_{w}-V_{z}+[V, U]=0 .
$$

A direct computation shows that the equation (2.1.6) can be rephrased as follows:

$$
\left\{\begin{array}{l}
u_{z w}-2 R Q e^{-u}+\frac{1}{2} H^{2} e^{u}=0, \\
Q_{w}-\frac{1}{2} H_{z} e^{u}=0, \\
R_{z}-\frac{1}{2} H_{w} e^{u}=0 .
\end{array}\right.
$$


The first equation in (2.1.7) will be called the complex Gauss equation, and the second and third equations in (2.1.7) will be called the complex Codazzi equations.

From the discussion above we know that all holomorphic null immersions $\Psi$ : $\mathcal{M} \rightarrow \mathbb{C}^{3}$ satisfy the complex Gauss-Codazzi equations (2.1.7). We note that, setting $\alpha=F^{-1} d F=U d z+V d w$, the equations in (2.1.7) are equivalent to

$$
d \alpha+\frac{1}{2}[\alpha \wedge \alpha]=0
$$

Using the functions $u, Q, R$ and $H$ defined in (2.1.2) and (2.1.5), respectively, the symmetric quadratic form $I I:=-\langle d \Psi, d N\rangle$ can be represented as follows:

$$
I I:=-\langle d \Psi, d N\rangle=Q d z^{2}+e^{u} H d z d w+R d w^{2} .
$$

The symmetric quadratic form II is called the second fundamental form for a holomorphic null immersion $\Psi$. Then the complex mean curvature and the complex Gauss curvature for a holomorphic null immersion $\Psi$ are defined as follows.

Definition 1. Let $\Psi: \mathcal{M} \rightarrow \mathbb{C}^{3}$ be a holomorphic null immersion. Then the function $H=2 e^{-u}\left\langle\Psi_{z w}, N\right\rangle$ will be called the complex mean curvature of $\Psi$.

Remark 2.3. From the forms of the holomorphic metric $g$ defined in (2.1.2) and the second fundamental form $I I$ defined in (2.1.8), the equality $H=\frac{1}{2} \operatorname{Tr}\left(\tilde{I}^{-1} \cdot \widetilde{I I}\right)$ holds, where $\tilde{I}$ (resp. $\widetilde{I I}$ ) is the coefficient matrix of $g$ (resp. $I I$ ).

Definition 2. We retain the notation in Remark 2.3. Then the function $K=$ $\operatorname{det}\left(\tilde{I}^{-1} \cdot \widetilde{I I}\right)=H^{2}-4 e^{-2 u} Q R$ will be called the complex Gauss curvature of $\Psi$.

2.2. Complex CMC- and CGC-immersions in $\mathbb{C}^{3}$. In this subsection, we give characterizations of complex constant mean curvature immersions via loop groups (see Appendix A.2 for the definitions of loop groups). There is a useful formula representing complex CMC-immersions, which is a generalization of the Sym formula for CMC-immersions in $\mathbb{R}^{3}$; see also $[9$. There is also a formula for complex CGC-immersions given by the parallel holomorphic immersions of complex CMCimmersions with $H \in \mathbb{C}^{*}$.

The notions of a complex CMC-immersion and a CGC-immersion are defined analogous to the notions of a CMC-immersion and a CGC-immersion in $\mathbb{R}^{3}$; see also [10.

Definition 3. Let $\Psi: \mathcal{M} \rightarrow \mathbb{C}^{3}$ be a holomorphic null immersion, and let $H$ (resp. $\mathrm{K})$ be its complex mean curvature (resp. Gauss curvature). Then $\Psi$ is called a complex constant mean curvature (CMC for short) immersion (resp. a complex constant Gauss curvature (CGC for short) immersion) if $H$ (resp. K) is a complex constant.

Remark 2.4. Since we are interested in complexifications of CMC (resp. CGC) surfaces with nonzero mean curvature $H \in \mathbb{R}^{*}$ (resp. Gauss curvature $K \in \mathbb{R}^{*}$ ), from now on, we always assume that the complex mean curvature $H$ (resp. the complex Gauss curvature $K$ ) is a nonzero constant.

From [10], we quote the following characterizations of a complex CMC-immersion:

Lemma 2.5. Let $\mathcal{M}$ be a connected 2-dimensional Stein manifold, and let $\Psi$ : $\mathcal{M} \rightarrow \mathbb{C}^{3}(\cong \mathfrak{s l}(2, \mathbb{C}))$ be a holomorphic null immersion. Further, let $Q, R, H$ and 
$N$ be the complex functions defined in (2.1.5) and the Gauss map defined in (2.1.3), respectively. Then the following statements are equivalent:

(1) $H$ is a nonzero constant.

(2) $Q$ depends only on $z$ and $R$ depends only on $w$.

(3) $N_{z w}=\rho N$, for some holomorphic function $\rho: \mathcal{M} \rightarrow \mathbb{C}$.

(4) There exists $\tilde{F}(z, w, \lambda) \in \Lambda S L(2, \mathbb{C})_{\sigma}$ such that

$$
\tilde{F}(z, w, \lambda)^{-1} d \tilde{F}(z, w, \lambda)=\tilde{U} d z+\tilde{V} d w
$$

where

$$
\left\{\begin{array}{c}
\tilde{U}=\left(\begin{array}{cc}
\frac{1}{4} u_{z} & -\frac{1}{2} \lambda^{-1} H e^{u / 2} \\
\lambda^{-1} Q e^{-u / 2} & -\frac{1}{4} u_{z}
\end{array}\right), \\
\tilde{V}=\left(\begin{array}{cc}
-\frac{1}{4} u_{w} & -\lambda R e^{-u / 2} \\
\frac{1}{2} \lambda H e^{u / 2} & \frac{1}{4} u_{w}
\end{array}\right),
\end{array}\right.
$$

and $\tilde{F}(z, w, \lambda=1)=F(z, w)$ is the moving frame of $\Psi$ in (2.1.4).

The $\tilde{F}(z, w, \lambda)$ defined in (4) of Lemma 2.5 is called the complex extended framing of a complex CMC-immersion $\Psi$. From now on, for simplicity, the symbol $F(z, w, \lambda)$ (resp. $U(z, w, \lambda)$ or $V(z, w, \lambda))$ is used instead of $\tilde{F}(z, w, \lambda)$ (resp. $\tilde{U}(z, w, \lambda)$ or $\tilde{V}(z, w, \lambda))$.

There is an immersion formula for a complex CMC-immersion using the complex extended framing $F(z, w, \lambda)$ for a complex CMC-immersion $\Psi$, the so-called "Sym formula"; see [10. We show a similar immersion formula for a complex CGCimmersion using the same complex extended framing $F(z, w, \lambda)$ of a complex CMCimmersion $\Psi$.

Theorem 2.6. Let $F(z, w, \lambda)$ be the complex extended framing of some complex $C M C$-immersion defined as in Lemma 2.5, and let $H$ be its nonzero complex constant mean curvature. We set

$$
\left\{\begin{array}{l}
\Psi=-\frac{1}{2 H}\left(i \lambda \partial_{\lambda} F(z, w, \lambda) \cdot F(z, w, \lambda)^{-1}+\frac{i}{2} F(z, w, \lambda) \sigma_{3} F(z, w, \lambda)^{-1}\right), \\
\Phi=-\frac{1}{2 H}\left(i \lambda \partial_{\lambda} F(z, w, \lambda) \cdot F(z, w, \lambda)^{-1}\right)
\end{array}\right.
$$

where $\sigma_{3}$ has been defined in (2.0.2). Then $\Psi$ (resp. $\left.\Phi\right)$ is, for every $\lambda \in \mathbb{C}^{*}$, a complex constant mean curvature immersion (resp. complex constant Gaussian curvature immersion, possibly degenerate) in $\mathbb{C}^{3}$ with complex mean curvature $H \in$ $\mathbb{C}^{*}$ (resp. complex Gauss curvature $K=4 H^{2} \in \mathbb{C}^{*}$ ), and the Gauss map of $\Psi$ (resp. $\Phi)$ can be described by $\frac{i}{2} F(z, w, \lambda) \sigma_{3} F(z, w, \lambda)^{-1}$.

Proof. The proof for complex CMC-immersions follows from [10. We show that the second formula $\Phi$ in (2.2.1) defines a complex CGC-immersion. Let $\Phi$ be a map in the second formula in (2.2.1). Let $N=\frac{i}{2} F(z, w, \lambda) \sigma_{3} F(z, w, \lambda)^{-1}$ be the complex Gauss map for $\Psi$. Since $\langle N, N\rangle=1$ and the relation $\Phi=\Psi+\frac{1}{2 H} N$ holds for the formulas in (2.2.1), $N$ is also the Gauss map for $\Phi$, i.e., $\left\langle\Phi_{z}, N\right\rangle=$ $\left\langle\Phi_{w}, N\right\rangle=0$. We also denote the Gauss map for $\Phi$ by $N$. 
We then compute the holomorphic metric $g$ and the second fundamental form II for the holomorphic map $\Phi$. Using the bilinear form in (2.1.1), we have

$$
\left\{\begin{array}{l}
\left\langle\Phi_{z}, \Phi_{z}\right\rangle=-2 \operatorname{Tr}\left(\Phi_{z} \cdot \Phi_{z}\right)=\frac{\lambda^{2}}{2 H^{2}} \operatorname{Tr}\left(\operatorname{Ad}(F) U_{\lambda}^{2}\right) \\
\left\langle\Phi_{w}, \Phi_{w}\right\rangle=-2 \operatorname{Tr}\left(\Phi_{w} \cdot \Phi_{w}\right)=\frac{\lambda^{2}}{2 H^{2}} \operatorname{Tr}\left(\operatorname{Ad}(F) V_{\lambda}^{2}\right)
\end{array}\right.
$$

where $U$ and $V$ are defined in Lemma 2.5 and the subscript $z$ (resp. $w$ or $\lambda$ ) denotes the partial derivative with respect to $z$ (resp. $w$ or $\lambda$ ). Since the trace of a matrix is invariant under the map $\operatorname{Ad}(F)$, and using the form of $U$ in Lemma 2.5. we have

$$
\left\{\begin{array}{l}
\left\langle\Phi_{z}, \Phi_{z}\right\rangle=\frac{\lambda^{2}}{2 H^{2}} \operatorname{Tr}\left(U_{\lambda}^{2}\right)=-\frac{1}{2 H^{2}} \lambda^{-2} H Q, \\
\left\langle\Phi_{w}, \Phi_{w}\right\rangle=\frac{\lambda^{2}}{2 H^{2}} \operatorname{Tr}\left(V_{\lambda}^{2}\right)=-\frac{1}{2 H^{2}} \lambda^{2} H R .
\end{array}\right.
$$

Again using the invariace of trace of a matrix under the map $\operatorname{Ad}(F)$ and the forms of $U$ and $V$ in Lemma 2.5. we have

$$
\begin{aligned}
\left\langle\Phi_{z}, \Phi_{w}\right\rangle & =-2 \operatorname{Tr}\left(-\frac{i \lambda}{2 H} \operatorname{Ad}(F) U_{\lambda} \times-\frac{i \lambda}{2 H} \operatorname{Ad}(F) V_{\lambda}\right) \\
& =\frac{1}{2 H^{2}}\left(\frac{1}{4} H^{2} e^{u}+Q R e^{-u}\right) .
\end{aligned}
$$

Therefore, we have the following first fundamental form for the holomorphic map $\Phi$ :

$$
g=(d z, \quad d w)\left(\begin{array}{cc}
-\frac{1}{2 H^{2}} \lambda^{-2} H Q & \frac{1}{2 H^{2}}\left(\frac{1}{4} H^{2} e^{u}+Q R e^{-u}\right) \\
\frac{1}{2 H^{2}}\left(\frac{1}{4} H^{2} e^{u}+Q R e^{-u}\right) & -\frac{1}{2 H^{2}} \lambda^{2} H R
\end{array}\right)\left(\begin{array}{l}
d z \\
d w
\end{array}\right) .
$$

Let $\tilde{I}$ denote the coefficient matrix for $g$ in (2.2.2). Then it is easy to verify that $\operatorname{det} \tilde{I}=-\left(\frac{1}{4} H^{2} e^{u}-Q R e^{-u}\right)^{2} /\left(4 H^{4}\right)$. Thus the holomorphic map $\Phi$ actually defines a holomorphic immersion under the condition $e^{2 u} \neq 4 H^{-2} Q R$.

Next, the second fundamental form $I I$ for $\Phi$ is computed as follows. Again using the invariace of the trace of a matrix under the map $\operatorname{Ad}(F),\left\langle\Phi_{z}, N_{z}\right\rangle$ is computed as follows:

$$
\begin{aligned}
\left\langle\Phi_{z}, N_{z}\right\rangle & =-2 \operatorname{Tr}\left(-\frac{i \lambda}{2 H} \operatorname{Ad}(F) U_{\lambda} \times \frac{i}{2} \operatorname{Ad}(F)\left[U, \sigma_{3}\right]\right) \\
& =-\frac{1}{2 H} \operatorname{Tr}\left(U_{\lambda} \cdot\left[U, \sigma_{3}\right]\right) .
\end{aligned}
$$

From the form of $U$ in Lemma 2.5, we have $\left\langle\Phi_{z}, N_{z}\right\rangle=0$. A similar argument holds for $\left\langle\Phi_{w}, N_{w}\right\rangle$, where $U$ is replaced by $V$. Thus we have $\left\langle\Phi_{w}, N_{w}\right\rangle=0$. Again using the invariance of the trace of a matrix under the map $\operatorname{Ad}(F)$ and the form of $U$ in Lemma 2.5, we obtain

$$
\begin{aligned}
\left\langle\Phi_{z}, N_{w}\right\rangle & =-2 \operatorname{Tr}\left(-\frac{i \lambda}{2 H} \operatorname{Ad}(F) U_{\lambda} \times \frac{i}{2} \operatorname{Ad}(F)\left[V, \sigma_{3}\right]\right) \\
& =-\frac{1}{2 H}\left(\frac{1}{2} H^{2} e^{u}-2 Q R e^{-u}\right) .
\end{aligned}
$$


Since $N$ is the Gauss map of $\Phi$, i.e., $\left\langle\Phi_{z}, N\right\rangle=\left\langle\Phi_{w}, N\right\rangle=0$ and $\langle N, N\rangle=1$, we obtain $\left\langle\Phi_{z}, N_{w}\right\rangle=-\left\langle\Phi_{w z}, N\right\rangle=\left\langle\Phi_{w}, N_{z}\right\rangle$.

Finally, the second fundamental form $I I$ for $\Phi$ has the following form:

$$
I I=\left(\begin{array}{ll}
d z, & d w
\end{array}\right)\left(\begin{array}{cc}
0 & \frac{1}{2 H}\left(\frac{1}{2} H^{2} e^{u}-2 Q R e^{-u}\right) \\
\frac{1}{2 H}\left(\frac{1}{2} H^{2} e^{u}-2 Q R e^{-u}\right) & 0
\end{array}\right)\left(\begin{array}{l}
d z \\
d w
\end{array}\right) .
$$

Let us denote the coefficient matrix of $I I$ by $\widetilde{I I}$. Then, using (2.2.2) and (2.2.3), the complex Gauss curvature $K$ for $\Phi$ is computed as

$$
K=\operatorname{det}\left(\tilde{I}^{-1} \cdot \widetilde{I I}\right)=4 H^{2} \in \mathbb{C}^{*} .
$$

This completes the proof.

Since the Gauss maps of a complex CMC-immersion $\Psi$ and the corresponding complex CGC-immersion $\Phi$ are the same, which is $N=\frac{i}{2} F(z, w, \lambda) \sigma_{3} F(z, w, \lambda)^{-1}$, we have the following corollary:

Corollary 2.7. Let $\Psi$ be a complex CMC-immersion, and let $H$ (resp. $u, Q$ and $R$ ) be its nonzero constant mean curvature (resp. the functions defined in (2.1.2) and Theorem 2.2). Moreover, let us assume $e^{2 u} \neq 4 H^{-2} Q R$. Then there exists the parallel complex CGC-immersion with Gauss curvature $K=4 H^{2} \in \mathbb{C}^{*}$.

2.3. Real forms of $\Lambda \mathfrak{s t}(2, \mathbb{C})_{\sigma}$. In this subsection, we give the classification of real forms for the twisted $\mathfrak{s l}(2, \mathbb{C})$ loop algebra $\Lambda \mathfrak{s l}(2, \mathbb{C})_{\sigma}$; see Appendix $\mathrm{A}$ for the notation and the definitions of loop algebras.

First we recall the basic facts about real forms for complex Kac-Moody Lie algebras. Let $\mathfrak{g}$ be a Kac-Moody Lie algebra over $\mathbb{C}$. Then a Lie subalgebra $\mathfrak{g}_{\mathbb{R}} \subset \mathfrak{g}$ over $\mathbb{R}$ will be called the real form of $\mathfrak{g}$ if there is an isomorphism between $\mathfrak{g}$ and the complexification $\mathfrak{g}_{\mathbb{R}} \otimes \mathbb{C}$. We note that all real forms of $\mathfrak{g}$ correspond to semilinear involutions of $\mathfrak{g}$ (see, for example, 1]), i.e., each real form is defined by an automorphism $\rho$ of $\mathfrak{g}$ such that

$$
\left\{\begin{array}{l}
\rho^{2}=\mathrm{id}, \\
\rho(\ell x)=\bar{\ell} \rho(x) \text { for } \ell \in \mathbb{C} .
\end{array}\right.
$$

Let $\mathfrak{h}$ denote the standard Cartan subalgebra of $\mathfrak{g}$, which is a maximal ad $(\mathfrak{g})$ diagonalizable subalgebra of $\mathfrak{g}$. Let $\Delta$ be the corresponding root system, and let $\mathfrak{g}_{\alpha}$ denote the root space corresponding to $\alpha$ in $\Delta$. Then the root space decomposition for $\mathfrak{g}$ is as follows:

$$
\mathfrak{g}=\mathfrak{h} \oplus\left(\bigoplus_{\alpha \in \Delta} \mathfrak{g}_{\alpha}\right)
$$

It is known that $\Delta$ can be decomposed as $\Delta=\Delta^{+} \cup \Delta^{-}$, where $\Delta^{+}$(resp. $\Delta^{-}$) is the set of positive (resp. negative) roots.

Then a subalgebra of $\mathfrak{g}$ is said to be a Borel subalgebra if it is a maximal completely solvable subalgebra. And the standard positive (resp. negative) Borel subalgebra $\mathfrak{b}^{+}\left(\right.$resp. $\left.\mathfrak{b}^{-}\right)$of $\mathfrak{g}$ is defined as follows:

$$
\mathfrak{b}^{ \pm}=\mathfrak{h} \oplus\left(\bigoplus_{\beta \in \Delta^{ \pm}} \mathfrak{g}_{\beta}\right)
$$


If a linear or semi-linear automorphism $\rho$ for $\mathfrak{g}$ transforms a Borel subalgebra into a Borel subalgebra of the same (resp. opposite) sign, then $\rho$ is said to be the first kind (resp. second kind).

We now give definitions of the almost split real forms and the almost compact real forms of $\mathfrak{g}$.

Definition 4. Let $\mathfrak{g}$ be a Kac-Moody Lie algebra over $\mathbb{C}$, and let $\mathfrak{g}_{\mathbb{R}}$ be a real form of $\mathfrak{g}$. Moreover, let $\rho$ be the semi-linear involution corresponding to $\mathfrak{g}_{\mathbb{R}}$. Then the real form $\mathfrak{g}_{\mathbb{R}}$ is called almost split (resp. almost compact) if the corresponding semi-linear involution $\rho$ is of the first kind (resp. second kind).

It is clear that the real subalgebra of $\mathfrak{g}$ generated by $\left\{\mathfrak{h}, e_{j}, f_{j} ; j=1,2, \ldots, n\right\}$ (the Cartan subalgebra and the Chevalley generators of the Kac-Moody Lie algebra $\mathfrak{g}$, see Appendix A.11 is an almost split real form, which is called the standard split form. The corresponding semi-linear involution of the first kind $\sigma_{n}^{\prime}$ is called the standard normal semi-involution of $\mathfrak{g}$. The map $e_{j} \mapsto-f_{j}, f_{j} \mapsto-e_{j}$ and $h \mapsto-h$, $h \in \mathfrak{h}$ for $\left\{\mathfrak{h}, e_{j}, f_{j} ; j=1,2, \ldots, n\right\}$ can be extended to an involution $\omega$ of $\mathfrak{g}$. The $\omega$ is called the Cartan involution of $\mathfrak{g}$. It is known that the standard normal semi-involution and the Cartan involution commute.

Definition 5. Let $\omega$ and $\sigma_{n}^{\prime}$ be the Cartan involution and the standard normal semi-involution, respectively, and let $\omega^{\prime}$ be $\omega^{\prime}=\sigma_{n}^{\prime} \omega=\omega \sigma_{n}^{\prime}$. Then $\omega^{\prime}$ is called the standard Cartan semi-involution, and the corresponding almost compact real form is called the standard compact form. Moreover, a conjugation of $\omega^{\prime}$ is called a Cartan semi-involution.

We quote the following theorem about the real forms of Kac-Moody Lie algebras [3], 4.

Theorem 2.8 (Theorem 4.4 in [3, Proposition 2.9 in 4]). Let us consider the following:

(1) The semi-linear involutions $\rho$ of $\mathfrak{g}$ of the second kind (resp. the first kind).

(2) The involutions $\theta$ of $\mathfrak{g}$ of the first kind (resp. the second kind).

(3) The relation $\rho \approx \theta$ if and only if

(a) $\omega^{\prime}=\theta \rho=\rho \theta$ is a Cartan semi-involution,

(b) $\theta$ and $\rho$ stabilize the same Cartan subalgebra $\mathfrak{h}$,

(c) $\mathfrak{h}$ is contained in a minimal $\rho$-stable positive parabolic subalgebra.

Then the relation induces a bijection between the conjugacy classes under Aut $(\mathfrak{g})$ of semi-linear involutions of the second kind (resp. the first kind) and conjugacy classes of involutions of the first kind (resp. the second kind).

We also quote the following theorem about the classification of the involutions of the affine Kac-Moody Lie algebra of $A_{1}^{(1)}$ type [17.

Theorem 2.9 (Theorem 3 in [17]). All involutions on the affine Kac-Moody Lie algebra of type $A_{1}^{(1)}$ are given as follows:

(a) $e_{1} \longmapsto-e_{1}, \quad f_{1} \longmapsto-f_{1}, \quad e_{2} \longmapsto-e_{2}, \quad f_{2} \longmapsto-f_{2}$,

$\left(\mathrm{a}^{\prime}\right) e_{1} \longmapsto e_{2}, \quad f_{1} \longmapsto f_{2}, \quad e_{2} \longmapsto e_{1}, \quad f_{2} \longmapsto f_{1}$,

(b) $e_{1} \longmapsto e_{1}, \quad f_{1} \longmapsto f_{1}, \quad e_{2} \longmapsto-\frac{1}{2}\left[\left[f_{2}, f_{1}\right], f_{1}\right], \quad f_{2} \longmapsto-\frac{1}{2}\left[\left[e_{2}, e_{1}\right], e_{1}\right]$, 


$$
\begin{aligned}
& \left(\mathrm{b}^{\prime}\right) e_{1} \longmapsto-e_{1}, \quad f_{1} \longmapsto-f_{1}, \quad e_{2} \longmapsto \frac{1}{2}\left[\left[f_{2}, f_{1}\right], f_{1}\right], \quad f_{2} \longmapsto \frac{1}{2}\left[\left[e_{2}, e_{1}\right], e_{1}\right], \\
& \left(\mathrm{b}^{\prime \prime}\right) e_{1} \longmapsto f_{2}, \quad f_{1} \longmapsto e_{2}, \quad e_{2} \longmapsto f_{1}, \quad f_{2} \longmapsto e_{1}, \\
& (\mathrm{c}) e_{1} \longmapsto e_{1}, \quad f_{1} \longmapsto f_{1}, \quad e_{2} \longmapsto-e_{2}, \quad f_{2} \longmapsto-f_{2},
\end{aligned}
$$

where $e_{j}, f_{j}$ for $j \in\{1,2\}$ are the Chevalley generators of the affine Kac-Moody Lie algebra of $A_{1}^{(1)}$ type. Moreover, the cases (a), ( $\left.\mathrm{a}^{\prime}\right)$ and (c) (resp. (b), ( $\left.\mathrm{b}^{\prime}\right)$ and $\left(\mathrm{b}^{\prime \prime}\right)$ ) are involutions of the first kind (resp. the second kind).

It is well known that the twice central extensions of the untwisted $\mathfrak{s l}(2, \mathbb{C})$ loop algebra $\Lambda \mathfrak{s l}(2, \mathbb{C})$ is an affine Kac-Moody Lie algebra of $A_{1}^{(1)}$ type (see Theorem A.1). It is also known that the twisted $\mathfrak{s l}(2, \mathbb{C})$ loop algebra $\Lambda \mathfrak{s l}(2, \mathbb{C})_{\sigma}$ and the untwisted $\mathfrak{s l}(2, \mathbb{C})$ loop algebra $\Lambda \mathfrak{s l}(2, \mathbb{C})$ are isomorphic by the following map from $\Lambda \mathfrak{s l}(2, \mathbb{C})$ to $\Lambda \mathfrak{s l}(2, \mathbb{C})_{\sigma}$; see also [16]:

$$
g(\lambda) \in \Lambda \mathfrak{s l}(2, \mathbb{C}) \mapsto \operatorname{Ad}\left(\begin{array}{cc}
\sqrt{\lambda} & 0 \\
0 & \sqrt{\lambda}^{-1}
\end{array}\right) g\left(\lambda^{2}\right) \in \Lambda \mathfrak{s l}(2, \mathbb{C})_{\sigma} .
$$

Therefore we have the following classification of all real forms for $\Lambda \mathfrak{s l}(2, \mathbb{C})_{\sigma}$.

Theorem 2.10. Let $\mathfrak{c}_{j}$ for $j \in\{1,2,3,4\}$ be the following involutions on $\Lambda \mathfrak{s l}(2, \mathbb{C})_{\sigma}$ :

$$
\begin{array}{ll}
\mathfrak{c}_{1}: g(\lambda) \mapsto-\overline{g(-1 / \bar{\lambda})}^{t}, & \mathfrak{c}_{2}: g(\lambda) \mapsto \overline{g(-1 / \bar{\lambda})} \\
\mathfrak{c}_{3}: g(\lambda) \mapsto-\overline{g(1 / \bar{\lambda})}^{t}, & \mathfrak{c}_{4}: g(\lambda) \mapsto-\operatorname{Ad}\left(\begin{array}{cc}
1 / \sqrt{i} & 0 \\
0 & \sqrt{i}
\end{array}\right) \overline{g(i / \bar{\lambda})}^{t},
\end{array}
$$

where $g(\lambda) \in \Lambda \mathfrak{s l}(2, \mathbb{C})_{\sigma}$. Then, the almost compact real forms of $\Lambda \mathfrak{s l}(2, \mathbb{C})_{\sigma}$ are the following real Lie subalgebras of $\Lambda \mathfrak{s l}(2, \mathbb{C})_{\sigma}$ :

$$
\Lambda \mathfrak{s l}(2, \mathbb{C})_{\sigma}^{(\mathfrak{c}, j)}=\left\{g(\lambda) \in \Lambda \mathfrak{s l}(2, \mathbb{C})_{\sigma} \mid \mathfrak{c}_{j} \circ g(\lambda)=g(\lambda)\right\} \text { for } j \in\{1,2,3,4\} .
$$

Theorem 2.11. Let $\mathfrak{s}_{j}$ for $j \in\{1,2,3\}$ be the following involutions on $\Lambda \mathfrak{s l}(2, \mathbb{C})_{\sigma}$ :

$$
\begin{array}{ll}
\mathfrak{s}_{1}: g(\lambda) \mapsto-\overline{g(-\bar{\lambda})}^{t}, & \mathfrak{s}_{2}: g(\lambda) \mapsto \overline{g(-\bar{\lambda})}, \\
\mathfrak{s}_{3}: g(\lambda) \mapsto-\operatorname{Ad}\left(\begin{array}{cc}
\lambda & 0 \\
0 & \lambda^{-1}
\end{array}\right) \overline{g(\bar{\lambda})}^{t}, &
\end{array}
$$

where $g(\lambda) \in \Lambda \mathfrak{s l}(2, \mathbb{C})_{\sigma}$. Then, the almost split real forms of $\Lambda \mathfrak{s l}(2, \mathbb{C})_{\sigma}$ are the following real Lie subalgebras of $\Lambda \mathfrak{s l}(2, \mathbb{C})_{\sigma}$ :

$$
\Lambda \mathfrak{s l}(2, \mathbb{C})_{\sigma}^{(\mathfrak{s}, j)}=\left\{g(\lambda) \in \Lambda \mathfrak{s l}(2, \mathbb{C})_{\sigma} \mid \mathfrak{s}_{j} \circ g(\lambda)=g(\lambda)\right\} \text { for } j \in\{1,2,3\} .
$$

Proof. Since all linear involutions of the first kind and the second kind for the affine Kac-Moody Lie algebra of $A_{1}^{(1)}$ type are classified in Theorem 2.9, and using Theorem 2.8 all semi-linear involutions $\rho$ of the first kind (resp. the second kind) can be represented as follows:

$$
\rho=\omega^{\prime} \theta,
$$

where $\omega^{\prime}$ is the Cartan semi-involution and $\theta$ is the linear involution of the second kind (resp. the first kind). Noting that the identity map is also the trivial involution of the first kind, we have the four classes of semi-linear involutions of the second kind and the three classes of semi-linear involutions of the first kind. Since the affine Kac-Moody Lie algebra of $A_{1}^{(1)}$ type can be realized by the twice central extensions of the loop algebra $\Lambda \mathfrak{s l}(2, \mathbb{C}$ ) (see Theorem A.1), the real forms of the loop algebra $\Lambda \mathfrak{s l}(2, \mathbb{C})$ are derived. Finally, using the isomorphism (2.3.2), the real forms of $\Lambda \mathfrak{s l}(2, \mathbb{C})$ are transformed into the real forms of $\Lambda \mathfrak{s l}(2, \mathbb{C})_{\sigma}$, which are obtained in (2.3.3) and 2.3.5) by a direct calculation. This completes the proof. 
Remark 2.12. The identification (2.3.2) implies, in the untwisted setting, the involution $\mathfrak{s}_{3}$ can be rephrased as follows:

$$
\mathfrak{s}_{3}: g(\lambda) \mapsto-\bar{g}^{t}{ }^{t} \text { for } g(\lambda) \in \Lambda \mathfrak{s l}(2, \mathbb{C}) .
$$

From now on we use the involution (2.3.7) instead of the original involution $\mathfrak{s}_{3}$ in (2.3.5).

\section{Real forms of COMPlex CGC-IMmersions}

In this section, we show one of the main theorems in this paper (Theorem 3.3), which is the classification of "integrable surfaces" obtained from all the real forms of the twisted $\mathfrak{s l}(2, \mathbb{C})$ loop algebra $\Lambda \mathfrak{s l}(2, \mathbb{C})_{\sigma}$.

3.1. Integrable surfaces as real forms of complex CGC-immersions. Let $F(z, w, \lambda) \in \Lambda S L(2, \mathbb{C})_{\sigma}$ be the complex extended framing of some complex CGCimmersion $\Phi$, and let $\alpha(z, w, \lambda)=F(z, w, \lambda)^{-1} d F(z, w, \lambda)$ be the Maurer-Cartan form of $F(z, w, \lambda)$. From the forms of $U$ and $V$ defined as in Lemma 2.5. we set $\alpha_{i}(i \in\{-1,0,1\})$ as follows:

$$
\alpha(z, w, \lambda)=F^{-1} d F=U d z+V d w=\lambda^{-1} \alpha_{-1}+\alpha_{0}+\lambda \alpha_{1},
$$

where

$$
\left\{\begin{array}{c}
\alpha_{-1}=\left(\begin{array}{cc}
0 & -\frac{1}{2} H e^{u / 2} d z \\
Q e^{-u / 2} d z & 0
\end{array}\right) \\
\alpha_{0}=\left(\begin{array}{cc}
\frac{1}{4} u_{z} d z-\frac{1}{4} u_{w} d w & 0 \\
0 & -\frac{1}{4} u_{z} d z+\frac{1}{4} u_{w} d w
\end{array}\right) \\
\alpha_{1}=\left(\begin{array}{cc}
0 & -R e^{-u / 2} d w \\
\frac{1}{2} H e^{u / 2} d w & 0
\end{array}\right) .
\end{array}\right.
$$

We denote the space of $\Lambda \mathfrak{s l}(2, \mathbb{C})_{\sigma}$ valued 1 -forms by $\Omega\left(\Lambda \mathfrak{s l}(2, \mathbb{C})_{\sigma}\right)$. Similar to the involutions in Theorem 2.10 (resp. Theorem 2.11), we define the involutions $\tilde{\mathfrak{c}}_{j}$ (resp. $\left.\tilde{\mathfrak{s}}_{j}\right)$ for $g(\lambda) \in \Omega\left(\Lambda \mathfrak{s l}(2, \mathbb{C})_{\sigma}\right)$ as follows:

$$
\left\{\begin{array} { l } 
{ \tilde { \mathfrak { c } } _ { 1 } : g ( \lambda ) \mapsto - \overline { g ( - 1 / \overline { \lambda } } ^ { t } , } \\
{ \tilde { \mathfrak { c } } _ { 2 } : g ( \lambda ) \mapsto \overline { ( - 1 / \overline { \lambda } ) } , } \\
{ \tilde { \mathfrak { c } } _ { 3 } : g ( \lambda ) \mapsto - \overline { g ( 1 / \overline { \lambda } ) ^ { t } , } } \\
{ \tilde { \mathfrak { c } } _ { 4 } : g ( \lambda ) \mapsto - \operatorname { A d } ( \begin{array} { c c } 
{ 1 / \sqrt { i } } & { 0 } \\
{ 0 } & { \sqrt { i } }
\end{array} ) \overline { g ( i / \overline { \lambda } ) } ^ { t } , }
\end{array} \quad \left\{\begin{array}{l}
\tilde{\mathfrak{s}}_{1}: g(\lambda) \mapsto-\overline{g(-\bar{\lambda})^{t}} \\
\tilde{\mathfrak{s}}_{2}: g(\lambda) \mapsto \overline{g(-\bar{\lambda})}, \\
\tilde{\mathfrak{s}}_{3}: g(\lambda) \mapsto-\overline{g(\bar{\lambda})}^{t} .
\end{array}\right.\right.
$$

Then the real forms of $\Omega\left(\Lambda \mathfrak{s l}(2, \mathbb{C})_{\sigma}^{(\mathfrak{c}, j)}\right)$ are defined as follows:

$$
\begin{aligned}
& \Omega\left(\Lambda \mathfrak{s l}(2, \mathbb{C})_{\sigma}^{(\mathfrak{c}, j)}\right)=\left\{g \in \Omega\left(\Lambda \mathfrak{s l}(2, \mathbb{C})_{\sigma}\right) \mid \tilde{\mathfrak{c}}_{j} \circ g(\lambda)=g(\lambda)\right\}, \\
& \Omega\left(\Lambda \mathfrak{s l}(2, \mathbb{C})_{\sigma}^{(\mathfrak{s}, j)}\right)=\left\{g \in \Omega\left(\Lambda \mathfrak{s l}(2, \mathbb{C})_{\sigma}\right) \mid \tilde{\mathfrak{s}}_{j} \circ g(\lambda)=g(\lambda)\right\} .
\end{aligned}
$$

From now on, for simplicity, we use the symbols $\mathfrak{c}_{j}$ and $\mathfrak{s}_{j}$ instead of $\tilde{\mathfrak{c}}_{j}$ and $\tilde{\mathfrak{s}}_{j}$, respectively. We now consider the following conditions on $\alpha(z, w, \lambda)$ :

- Almost compact cases $(C, j): \alpha(z, w, \lambda)$ is an element in the real form $\Omega\left(\Lambda \mathfrak{s l}(2, \mathbb{C})_{\sigma}^{(\mathfrak{c}, j)}\right)$. 
- Almost split cases $(S, j): \alpha(z, w, \lambda)$ is an element in the real form $\Omega\left(\Lambda \mathfrak{s l}(2, \mathbb{C})_{\sigma}^{(\mathfrak{s}, j)}\right)$.

A straightforward computation shows that the conditions above, which are the almost compact cases $(C, j)$ and the almost split cases $(S, j)$, are equivalent to the following equations for $\alpha_{i}(i \in\{-1,0,1\})$ :

$$
\begin{cases}\alpha_{0}=-\overline{\alpha_{0}} \text { and } \alpha_{ \pm j}={\overline{\alpha_{ \pm 1}}}^{t} & \text { for the }(C, 1) \text { or }(S, 1) \text { case } \\
\alpha_{0}=\overline{\alpha_{0}} \text { and } \alpha_{ \pm j}=-\overline{\alpha_{ \pm 1}} & \text { for the }(C, 2) \text { or }(S, 2) \text { case } \\
\alpha_{0}=-\overline{\alpha_{0}} \text { and } \alpha_{ \pm j}=-\overline{\alpha_{ \pm 1}} t & \text { for the }(C, 3) \text { or }(S, 3) \text { case } \\
\alpha_{0}=-\overline{\alpha_{0}} \text { and } \alpha_{-1}=i \operatorname{Ad}\left(\begin{array}{cc}
1 / \sqrt{i} & 0 \\
0 & \sqrt{i}
\end{array}\right) \bar{\alpha}_{1}^{t} & \text { for the }(C, 4) \text { case }\end{cases}
$$

where $j=-1$ (resp. $j=1$ ) if $\alpha$ satisfies one of the conditions for almost compact cases (resp. almost split cases). From the symmetry between $\alpha_{1}$ and $\alpha_{-1}$ for the almost compact cases and the symmetries on each of $\alpha_{1}$ and $\alpha_{-1}$ for the almost split cases, we obtain

$$
\left\{\begin{array}{l}
w=\bar{z} \text { for the almost compact cases }(C, j) \\
z=\bar{z} \text { and } w=\bar{w} \text { for the almost split cases }(S, j) .
\end{array}\right.
$$

Moreover, the following choices, which are unique up to constants, of $u, Q, R$ and $H$ for $\alpha(z, w, \lambda)$ in (3.1.2) give solutions for (3.1.5):

$$
\left\{\begin{array}{cl}
u \in \mathbb{R}, R=-\bar{Q}, H \in i \mathbb{R}^{*} & \text { for the }(C, 1) \text { case, } \\
u \in i \mathbb{R}, R=Q=-\frac{1}{2} \bar{H}, H \in \mathbb{C}^{*} & \text { for the }(C, 2) \text { case, } \\
u \in \mathbb{R}, R=\bar{Q}, H \in \mathbb{R}^{*} & \text { for the }(C, 3) \text { case, } \\
u \in \mathbb{R}, R=\bar{Q}, H \in i \mathbb{R}^{*} & \text { for the }(C, 4) \text { case, } \\
u \in i \mathbb{R}, Q=R=-\frac{1}{2} \bar{H}, H \in \mathbb{C}^{*} & \text { for the }(S, 1) \text { case, } \\
u \in \mathbb{R}, Q, R, H \in i \mathbb{R}^{*} & \text { for the }(S, 2) \text { case, } \\
u \in i \mathbb{R}, Q=R=\frac{1}{2} \bar{H}, H \in \mathbb{C}^{*} & \text { for the }(S, 3) \text { case. }
\end{array}\right.
$$

We denote loop groups whose loop algebras are $\Lambda \mathfrak{s l}(2, \mathbb{C})_{\sigma}^{(\mathfrak{c}, j)}$ and $\Lambda \mathfrak{s l}(2, \mathbb{C})_{\sigma}^{(\mathfrak{s}, j)}$ by

$$
\Lambda S L(2, \mathbb{C})_{\sigma}^{(\mathfrak{c}, j)} \text { for } j \in\{1,2,3,4\} \text { and } \Lambda S L(2, \mathbb{C})_{\sigma}^{(\mathfrak{s}, j)} \text { for } j \in\{1,2,3\}
$$

If the Maurer-Cartan form $\alpha=F^{-1} d F$ is in $\Omega\left(\Lambda \mathfrak{s l}(2, \mathbb{C})_{\sigma}^{(\mathfrak{c}, j)}\right)$ for $j \in\{1,2,3,4\}$ (resp. $\Omega\left(\Lambda \mathfrak{s l}(2, \mathbb{C})_{\sigma}^{(\mathfrak{s}, j)}\right)$ for $\left.j \in\{1,2,3\}\right)$, the corresponding complex extended framing $F$ is in $\Lambda S L(2, \mathbb{C})_{\sigma}^{(\mathfrak{c}, j)}$ (resp. $\Lambda S L(2, \mathbb{C})_{\sigma}^{(\mathfrak{s}, j)}$ ) under the initial condition $F\left(z_{*}, w_{*}, \lambda\right)=$ id with $\left(z_{*}, w_{*}\right)=\left(z_{*}, \bar{z}_{*}\right) \in \mathfrak{D}^{2}\left(\operatorname{resp} .\left(z_{*}, w_{*}\right)=\left(\bar{z}_{*}, \bar{w}_{*}\right) \in \mathfrak{D}^{2}\right)$. We denote the complex extended framing $F$ which is a loop in $\Lambda S L(2, \mathbb{C})_{\sigma}^{(\mathfrak{c}, j)}$ (resp. $\left.\Lambda S L(2, \mathbb{C})_{\sigma}^{(\mathfrak{s}, j)}\right)$ by $F^{(\mathfrak{c}, j)}\left(\operatorname{resp} . F^{(\mathfrak{s}, j)}\right)$. 
We now set the following formulas $\Phi^{(\mathfrak{c}, j)}$ for $j \in\{1,2,3,4\}$ (resp. $\Phi^{(\mathfrak{s}, j)}$ for $j \in\{1,2,3\})$ analogous to the second formula in (2.2.1):

$$
\Phi^{(\mathfrak{c}, j)}=-\left.\frac{1}{2|H|}\left(i \lambda \partial_{\lambda} F^{(\mathfrak{c}, j)}(z, \bar{z}, \lambda) \cdot F^{(\mathfrak{c}, j)}(z, \bar{z}, \lambda)^{-1}\right)\right|_{\lambda \in S^{1}} \text { for } j \in\{1,2,3\},
$$

$$
\Phi^{(\mathfrak{c}, 4)}=\left.\frac{1}{2}\left(F^{(\mathfrak{c}, 4)}(z, \bar{z}, \lambda)\left(\begin{array}{cc}
e^{q / 2} & 0 \\
0 & e^{-q / 2}
\end{array}\right)\left(F^{(\mathfrak{c}, 4)}(z, \bar{z}, \lambda)\right)^{*}\right)\right|_{\lambda \in S^{r}}
$$

$$
\Phi^{(\mathfrak{s}, j)}=-\left.\frac{1}{2|H|}\left(\lambda \partial_{\lambda} F^{(\mathfrak{s}, j)}(x, y, \lambda) \cdot F^{(\mathfrak{s}, j)}(x, y, \lambda)^{-1}\right)\right|_{\lambda \in \mathbb{R}^{*}} \text { for } j \in\{1,2,3\},
$$

where $\lambda=\exp (i t) \in S^{1}$ or $\lambda=\exp (q / 2+i t) \in S^{r}$ for 3.1.9) or 3.1.10) (resp. $\lambda= \pm \exp (t) \in \mathbb{R}^{*}$ for (3.1.11) $)$ with $t, q \in \mathbb{R}$, and where $*$ denotes $X^{*}=\bar{X}^{t}$ for $X \in M_{2 \times 2}(\mathbb{C})$. We note that $w=\bar{z}$ (resp. $z=\bar{z}=x \in \mathbb{R}$ and $w=\bar{w}=y \in \mathbb{R}$ ) for $\Phi^{(\mathfrak{c}, j)}\left(\operatorname{resp} . \Phi^{(\mathfrak{s}, j)}\right)$, from (3.1.6) $)$ Then, for each $\lambda \in S^{1}$ or $\lambda \in S^{r}\left(\right.$ resp. $\left.\lambda \in \mathbb{R}^{*}\right)$, the formula $\Phi^{(\mathfrak{c}, j)}$ (resp. $\Phi^{(\mathfrak{s}, j)}$ ) defines a map into one of the following spaces:

$$
\left\{\begin{array}{cl}
\mathfrak{s u}(1,1) \cong \mathbb{R}^{1,2} & \text { for the }(C, 1) \text { and }(S, 1) \text { cases } \\
\mathfrak{s l}_{*}(2, \mathbb{R}) \cong \mathbb{R}^{1,2} & \text { for the }(C, 2) \text { and }(S, 2) \text { cases } \\
\mathfrak{s u}(2) \cong \mathbb{R}^{3} & \text { for the }(C, 3) \text { and }(S, 3) \text { cases } \\
S L(2, \mathbb{C}) / S U(2) \cong H^{3} & \text { for the }(C, 4) \text { case }
\end{array}\right.
$$

where $\mathfrak{s l}_{*}(2, \mathbb{R})=\left\{g \in \mathfrak{s l}(2, \mathbb{C}) \mid g=\left(\begin{array}{cc}a & b \\ c & -a\end{array}\right), a \in \mathbb{R}, b, c \in i \mathbb{R}\right\}$, which is isomorphic to $\mathfrak{s l}(2, \mathbb{R})$. Here $\mathbb{R}^{1,2}$ and $\mathbb{R}^{3}$ can be identified with $\mathfrak{s u}(1,1), \mathfrak{s l}_{*}(2, \mathbb{R})$ and $\mathfrak{s u}(2)$ analogous to the identification (2.0.1). The Minkowski 4-space $\mathbb{R}^{3,1}$ can be identified with $\operatorname{Herm}(2):=\left\{X \in M_{2 \times 2}(\mathbb{C}) \mid \bar{X}^{t}=X\right\}$ via the map

$$
\left(x_{1}, x_{2}, x_{3}, x_{0}\right) \mapsto \frac{1}{2}\left(\begin{array}{cc}
x_{0}+x_{3} & x_{1}+i x_{2} \\
x_{1}-i x_{2} & x_{0}-x_{3}
\end{array}\right),
$$

then $H^{3} \subset \mathbb{R}^{3,1}$ can be identified with $\operatorname{Herm}(2)$ with the determinant $1 / 4$. Then the inner product for $\mathfrak{s u}(1,1) \cong \mathbb{R}^{1,2}\left(\right.$ resp. $\mathfrak{s l}_{*}(2, \mathbb{R}) \cong \mathbb{R}^{1,2}$ or $\left.\mathfrak{s u}(2) \cong \mathbb{R}^{3}\right)$ can be defined by $\langle a, b\rangle=-2 \operatorname{Tr}(a b)$ for $a, b \in \mathfrak{s u}(1,1)$ (resp. $a, b \in \mathfrak{s l}_{*}(2, \mathbb{R})$ or $a, b \in \mathfrak{s u}(2))$. The inner product for $\operatorname{Herm}(2) \cong \mathbb{R}^{3,1}$ can be defined by $\langle a, b\rangle=$ $-2 \operatorname{Tr}\left(a \sigma_{2} b^{t} \sigma_{2}\right)$ for $a, b \in \operatorname{Herm}(2)$, where $\sigma_{2}$ is defined in (2.0.2). From now on, we always assume that the spectral parameter $\lambda$ is in $S^{1}$ or $S^{r}$ for the almost compact cases and $\lambda$ is in $\mathbb{R}^{*}$ for the almost split cases, respectively.

Remark 3.1. For the $(C, 4)$ case, the complex Gauss equation in 2.1.7) can be reduced to the elliptic cosh-Gordon type equation by the choices of functions in (3.1.7). It is known that the Gauss equation for CMC surfaces with mean curvature $|H|<1$ in $H^{3}$ is the elliptic cosh-Gordon type equation; see 2. Therefore it is natural to use the Sym formula defined in (3.1.10) for the $(C, 4)$ case.

We denote the metrics for $\Phi^{(\mathfrak{c}, j)}$ by $g^{(\mathfrak{c}, j)}$ (resp. $\Phi^{(\mathfrak{s}, j)}$ by $g^{(\mathfrak{s}, j)}$ ), and also denote the coefficient matrices for the metrics $g^{(\mathfrak{c}, j)}$ by $\tilde{I}^{(\mathfrak{c}, j)}$ for $j \in\{1,2,3,4\}$ (resp. $g^{(\mathfrak{s}, j)}$ by $\tilde{I}^{(\mathfrak{s}, j)}$ for $\left.j \in\{1,2,3\}\right)$. 
Since $\lambda \in S^{1}$ or $S^{r}$ for the almost compact cases and $\lambda \in \mathbb{R}^{*}$ for the almost split cases, $\tilde{I}^{(\mathfrak{c}, j)}$ and $\tilde{I}^{(\mathfrak{s}, j)}$ are given as follows:

$$
\left\{\begin{array}{l}
\tilde{I}^{(\mathfrak{c}, j)}=\frac{1}{2|H|^{2}}\left(\begin{array}{cc}
\mathfrak{a}+\mathfrak{b}+\mathfrak{c} & i(\mathfrak{b}-\mathfrak{c}) \\
i(\mathfrak{b}-\mathfrak{c}) & \mathfrak{a}-\mathfrak{b}-\mathfrak{c}
\end{array}\right) \text { for } j \in\{1,2,3\} \\
\tilde{I}^{(\mathfrak{c}, 4)}=-H^{2} e^{u} \cosh ^{2}(q)\left(\begin{array}{cc}
1 & 0 \\
0 & 1
\end{array}\right) \\
\tilde{I}^{(\mathfrak{s}, j)}=\frac{1}{2|H|^{2}}\left(\begin{array}{cc}
-\mathfrak{b} & -\frac{1}{2} \mathfrak{a} \\
-\frac{1}{2} \mathfrak{a} & -\mathfrak{c}
\end{array}\right) \text { for } j \in\{1,2,3\},
\end{array}\right.
$$

where $\mathfrak{a}=H^{2} e^{u} / 2+2 Q R e^{-u}, \mathfrak{b}=-\lambda^{-2} H Q, \mathfrak{c}=-\lambda^{2} H R$ and $u, Q, R$ and $H$ are solutions defined in (3.1.7). Therefore it is easy to verify that the determinants of $\tilde{I}^{(\mathfrak{c}, j)}$ and $\tilde{I}^{(\mathfrak{s}, j)}$ are as follows:

$$
\left\{\begin{array}{l}
\operatorname{det} \widetilde{I}^{(\mathfrak{c}, j)}=\frac{1}{|H|^{4}}\left(\frac{1}{4} H^{2} e^{u}-Q R e^{-u}\right)^{2} \text { for } j \in\{1,2,3\}, \\
\operatorname{det} \widetilde{I}^{(\mathfrak{c}, 4)}=H^{4} e^{2 u} \cosh ^{4}(q) \\
\operatorname{det} \widetilde{I}^{(\mathfrak{s}, j)}=-\frac{1}{4|H|^{4}}\left(\frac{1}{4} H^{2} e^{u}-Q R e^{-u}\right)^{2} \text { for } j \in\{1,2,3\} .
\end{array}\right.
$$

From (3.1.13) one can verify that $\Phi^{(\mathfrak{c}, j)}$ and $\Phi^{(\mathfrak{s}, j)}$ actually define immersions if and only if $e^{u} \neq 4 H^{-2} Q R$ for $j \in\{1,2,3\}$, and for the $(C, 4)$ case, $\Phi^{(\mathfrak{c}, 4)}$ always defines an immersion. From (3.1.7), the immersions $\Phi^{(\mathfrak{c}, j)}$ for $j \in\{1,3,4\}$ and $\Phi^{(\mathfrak{s}, j)}$ for $j \in\{1,3\}$ are spacelike, and the immersions $\Phi^{(\mathfrak{c}, 2)}$ and $\Phi^{(\mathfrak{s}, 2)}$ are timelike.

Remark 3.2. Since we consider the Minkowski space $\mathbb{R}^{1,2}$ as the three-dimensional vector space $\left\{\left(x_{1}, x_{2}, x_{3}\right) \mid x_{j} \in \mathbb{R}\right\}$ endowed with the metric $g=d x_{1}^{2}-d x_{2}^{2}-d x_{3}^{2}$, the spacelike, timelike and lightlike vectors are $\langle a, a\rangle\langle 0,\langle b, b\rangle>0$ and $\langle c, c\rangle=0$ for $a, b, c \in \mathbb{R}^{1,2}$, respectively.

Let $N^{(\mathfrak{c}, j)}$ and $N^{(\mathfrak{s}, j)}$ be the following maps:

$$
\left\{\begin{aligned}
N^{(\mathfrak{c}, j)} & :=\frac{\ell}{2} \operatorname{Ad}\left(F^{(\mathfrak{c}, j)}\right) \sigma_{3}, \text { for } j \in\{1,2,3\}, \\
N^{(\mathfrak{c}, 4)} & :=\frac{1}{2} F^{(\mathfrak{c}, 4)}\left(\begin{array}{cc}
e^{q / 2} & 0 \\
0 & -e^{-q / 2}
\end{array}\right)\left(F^{(\mathfrak{c}, 4)}\right)^{*} \\
N^{(\mathfrak{s}, j)} & :=\frac{\ell}{2} \operatorname{Ad}\left(F^{(\mathfrak{s}, j)}\right) \sigma_{3} \text { for } j \in\{1,2,3\}
\end{aligned}\right.
$$

where $\ell$ is $i$ (resp. 1) for $j \in\{1,3\}$ (resp. $j=2$ ). It is clear that $N^{(\mathfrak{c}, j)}$ and $N^{(\mathfrak{s}, j)}$ are the Gauss maps of the immersions $\Phi^{(\mathfrak{c}, j)}$ and $\Phi^{(\mathfrak{s}, j)}$, respectively. The second fundamental forms $I I^{(\mathfrak{c}, j)}$ and $I I^{(\mathfrak{s}, j)}$ for the immersions $\Phi^{(\mathfrak{c}, j)}$ and $\Phi^{(\mathfrak{s}, j)}$ are defined by (see [21, page 107])

$$
\left\{\begin{array}{l}
I I^{(\mathfrak{c}, j)}=-\left\langle d \Phi^{(\mathfrak{c}, j)}, d N^{(\mathfrak{c}, j)}\right\rangle \text { for } j \in\{1,2,3,4\} \\
I I^{(\mathfrak{s}, j)}=-\left\langle d \Phi^{(\mathfrak{s}, j)}, d N^{(\mathfrak{s}, j)}\right\rangle \text { for } j \in\{1,2,3\} .
\end{array}\right.
$$

We denote the coefficient matrices of $I I^{(\mathfrak{c}, j)}$ by $\widetilde{I I}^{(\mathfrak{c}, j)}\left(\operatorname{resp} . I I^{(\mathfrak{s}, j)}\right.$ by $\left.\widetilde{I I}^{(\mathfrak{s}, j)}\right)$. A straightforward computation (see also the proof of Theorem 2.6) shows that the 
$\widetilde{I I}^{(\mathfrak{c}, j)}$ for $j \in\{1,2,3,4\}$ and $\widetilde{I I}^{(\mathfrak{s}, j)}$ for $j \in\{1,2,3\}$ are as follows:

$$
\left\{\begin{array}{l}
\widetilde{I I}^{(\mathfrak{c}, j)}=-\frac{2 i \ell}{|H|}\left(\frac{1}{4} H^{2} e^{u}-Q R e^{-u}\right)\left(\begin{array}{ll}
1 & 0 \\
0 & 1
\end{array}\right) \text { for } j \in\{1,2,3\}, \\
\widetilde{I I}^{(\mathfrak{c}, 4)}=\left(\begin{array}{cc}
\mathfrak{d}+2 \operatorname{Re} \mathfrak{e} & -2 \operatorname{Im} \mathfrak{e} \\
-2 \operatorname{Im} \mathfrak{e} & \mathfrak{d}-2 \operatorname{Re} \mathfrak{e}
\end{array}\right), \\
\widetilde{I I}^{(\mathfrak{s}, j)}=-\frac{\ell}{|H|}\left(\frac{1}{4} H^{2} e^{u}-Q R e^{-u}\right)\left(\begin{array}{ll}
0 & 1 \\
1 & 0
\end{array}\right) \text { for } j \in\{1,2,3\},
\end{array}\right.
$$

where $\mathfrak{d}=H^{2} e^{u} \cosh (q) \sinh (q)$ and $\mathfrak{e}=H Q \cosh (q) e^{-2 i t}$.

We recall that the Gauss curvatures $K^{(\mathfrak{c}, j)}$ and $K^{(\mathfrak{s}, j)}$ (resp. the mean curvature $H^{(\mathfrak{c}, 4)}$ ) of the immersions $\Phi^{(\mathfrak{c}, j)}$ and $\Phi^{(\mathfrak{s}, j)}$ for $j \in\{1,2,3\}$ (resp. $\Phi^{(\mathfrak{c}, 4)}$ ) are defined as follows (see also [22, page 157, (93)]):

$$
\left\{\begin{aligned}
K^{(\mathfrak{c}, j)}:= \pm \operatorname{det}\left(\tilde{I}^{(\mathfrak{c}, j)-1} \widetilde{I I}^{(\mathfrak{c}, j)}\right) \text { and } K^{(\mathfrak{s}, j)}:= \pm \operatorname{det}\left(\tilde{I}^{(\mathfrak{s}, j)-1} \widetilde{I I}^{(\mathfrak{s}, j)}\right) & \text { for } j \in\{1,2,3\}, \\
H^{(\mathfrak{c}, 4)}:=\frac{1}{2} \operatorname{Tr}\left(\tilde{I}^{(\mathfrak{c}, 4)-1} \widetilde{I I}^{(\mathfrak{c}, 4)}\right), &
\end{aligned}\right.
$$

where the plus sign (resp. the minus sign) has been chosen if the surface is in $\mathbb{R}^{3}$ or timelike in $\mathbb{R}^{1,2}$, i.e., $\Phi^{(\mathfrak{c}, j)}$ and $\Phi^{(\mathfrak{s}, j)}$ for $j \in\{2,3\}$ (resp. spacelike in $\mathbb{R}^{1,2}$, i.e., $\Phi^{(\mathfrak{c}, 1)}$ and $\left.\Phi^{(\mathfrak{s}, 1)}\right)$; see 22. Combining (3.1.13) with (3.1.15), we finally obtain

$$
\left\{\begin{array}{l}
K^{(\mathfrak{s}, 1)}=K^{(\mathfrak{s}, 2)}=K^{(\mathfrak{c}, 3)}=4|H|^{2}>0 \\
K^{(\mathfrak{c}, 1)}=K^{(\mathfrak{c}, 2)}=K^{(\mathfrak{s}, 3)}=-4|H|^{2}<0 \\
H^{(\mathfrak{c}, 4)}=\tanh (-q)
\end{array}\right.
$$

The above discussion is summarized in the following theorem:

Theorem 3.3. Let $F(z, w, \lambda)$ be the complex extended framing of some complex CGC-immersion $\Phi$. Then the following statements hold:

$(C, 1)$ If $F^{-1} d F$ is in $\Omega\left(\Lambda \mathfrak{s l}(2, \mathbb{C})_{\sigma}^{(\mathfrak{c}, 1)}\right)$, then for each $\lambda \in S^{1}$ the Sym formula in (3.1.9) defines a spacelike constant negative Gaussian curvature surface in $\mathbb{R}^{1,2}$.

$(C, 2)$ If $F^{-1} d F$ is in $\Omega\left(\Lambda \mathfrak{s l}(2, \mathbb{C})_{\sigma}^{(\mathfrak{c}, 2)}\right)$, then for each $\lambda \in S^{1}$ the Sym formula in (3.1.9) defines a timelike constant negative Gaussian curvature surface in $\mathbb{R}^{1,2}$.

$(C, 3)$ If $F^{-1} d F$ is in $\Omega\left(\Lambda \mathfrak{s l}(2, \mathbb{C})_{\sigma}^{(\mathfrak{c}, 3)}\right)$, then for each $\lambda \in S^{1}$ the Sym formula in (3.1.9) defines a constant positive Gaussian curvature surface in $\mathbb{R}^{3}$.

$(C, 4)$ If $F^{-1} d F$ is in $\Omega\left(\Lambda \mathfrak{s l}(2, \mathbb{C})_{\sigma}^{(\mathfrak{c}, 4)}\right)$, then for each $\lambda \in S^{r}$ the Sym formula in (3.1.10) defines a constant mean curvature surface with mean curvature $\left|H^{(\mathfrak{c}, 4)}\right|<1$ in $H^{3}$.

$(S, 1)$ If $F^{-1} d F$ is in $\Omega\left(\Lambda \mathfrak{s l}(2, \mathbb{C})_{\sigma}^{(\mathfrak{s}, 1)}\right)$, then for each $\lambda \in \mathbb{R}^{*}$ the Sym formula in (3.1.11) defines a spacelike constant positive Gaussian curvature surface in $\mathbb{R}^{1,2}$.

$(S, 2)$ If $F^{-1} d F$ is in $\Omega\left(\Lambda \mathfrak{s l}(2, \mathbb{C})_{\sigma}^{(\mathfrak{s}, 2)}\right)$, then for each $\lambda \in \mathbb{R}^{*}$ the Sym formula in (3.1.11) defines a timelike constant positive Gaussian curvature surface in $\mathbb{R}^{1,2}$. 
$(S, 3)$ If $F^{-1} d F$ is in $\Omega\left(\Lambda \mathfrak{s l}(2, \mathbb{C})_{\sigma}^{(\mathfrak{s}, 3)}\right)$, then for each $\lambda \in \mathbb{R}^{*}$ the Sym formula in (3.1.11) defines a constant negative Gaussian curvature surface in $\mathbb{R}^{3}$.

Definition 6. Let $F^{(\mathfrak{c}, j)}(z, \bar{z}, \lambda)$ for $j \in\{1,2,3,4\}$ (resp. $F^{(\mathfrak{s}, j)}(x, y, \lambda)$ for $j \in$ $\{1,2,3\})$ be the complex extended framings, which are elements in $\Lambda S L(2, \mathbb{C})_{\sigma}{ }^{(\mathfrak{c}, j)}$ (resp. $\left.\Lambda S L(2, \mathbb{C})_{\sigma}{ }^{(\mathfrak{s}, j)}\right)$. Then $F^{(\mathfrak{c}, j)}(z, w, \lambda)$ (resp. $\left.F^{(\mathfrak{s}, j)}(x, y, \lambda)\right)$ is called the extended framing for the immersion $\Phi^{(\mathfrak{c}, j)}\left(\operatorname{resp} . \Phi^{(\mathfrak{s}, j)}\right)$.

It is known that for three classes of surfaces in the above seven classes, there exist parallel constant mean curvature surfaces in $\mathbb{R}^{3}$ or $\mathbb{R}^{1,2}$; see also [13] and [14].

Corollary 3.4. We retain assumptions in Theorem 3.3 . Then we have the following:

$(C, 1 M)$ For the $(C, 1)$ case in Theorem 3.3 , there exists a parallel spacelike constant mean curvature surface with mean curvature $H^{(\mathfrak{c}, 1)}=|H|>0$ in $\mathbb{R}^{1,2}$.

$(C, 3 M)$ For the $(C, 3)$ case in Theorem 3.3 , there exists a parallel constant mean curvature surface with mean curvature $H^{(\mathfrak{c}, 3)}=|H|>0$ in $\mathbb{R}^{3}$.

$(S, 2 M)$ For the $(S, 2)$ case in Theorem 3.3 , there exists a parallel timelike constant mean curvature surface with mean curvature $H^{(\mathfrak{s}, 2)}=|H|>0$ in $\mathbb{R}^{1,2}$.

Proof. Let $\Phi^{(\mathfrak{c}, 1)}, \Phi^{(\mathfrak{c}, 3)}$ and $\Phi^{(\mathfrak{s}, 2)}$ be a spacelike constant negative Gaussian curvature surface in $\mathbb{R}^{1,2}$, a constant positive Gaussian curvature surface in $\mathbb{R}^{3}$ and a timelike constant positive Gaussian curvature surface in $\mathbb{R}^{1,2}$, as defined in Theorem 3.3. respectively. Let $N^{(\mathfrak{c}, 1)}, N^{(\mathfrak{c}, 3)}$ and $N^{(\mathfrak{s}, 2)}$ be the Gauss maps for $\Phi^{(\mathfrak{c}, 1)}, \Phi^{(\mathfrak{c}, 3)}$ and $\Phi^{(\mathfrak{s}, 2)}$ defined in (3.1.14), respectively. Then the parallel surfaces for $\Phi^{(\mathfrak{c}, 1)}$, $\Phi^{(\mathfrak{c}, 3)}$ and $\Phi^{(\mathfrak{s}, 2)}$ are defined by

$$
\left\{\begin{array}{l}
\Psi^{(\mathfrak{c}, j)}:=\Phi^{(\mathfrak{c}, j)}+\frac{1}{2|H|} N^{(\mathfrak{c}, j)} \text { for } j \in\{1,3\} \\
\Psi^{(\mathfrak{s}, 2)}:=\Phi^{(\mathfrak{s}, 2)}+\frac{1}{2|H|} N^{(\mathfrak{s}, 2)}
\end{array}\right.
$$

Then the first fundamental forms and the second fundamental forms for these immersions can be computed explicitly, and we can easily show that these immersions $\Psi^{(\mathfrak{c}, 1)}, \Psi^{(\mathfrak{c}, 3)}$ and $\Psi^{(\mathfrak{s}, 2)}$ define a spacelike constant mean curvature surface with mean curvature $H^{(\mathfrak{c}, 1)}=|H|$ in $\mathbb{R}^{1,2}$, a constant mean curvature surface with mean curvature $H^{(\mathfrak{c}, 3)}=|H|$ in $\mathbb{R}^{3}$ and a timelike constant mean curvature surface with mean curvature $H^{(\mathfrak{s}, 2)}=|H|$ in $\mathbb{R}^{1,2}$, respectively. This completes the proof.

Definition 7. The surfaces defined in Theorem 3.3 and Corollary 3.4 are called the integrable surfaces.

Remark 3.5. For the three classes of surfaces in Theorem 3.3, which are spacelike constant positive Gaussian curvature surfaces in $\mathbb{R}^{1,2}$, constant negative Gaussian curvature surfaces in $\mathbb{R}^{3}$ and timelike constant negative Gaussian curvature surfaces in $\mathbb{R}^{1,2}$, there never exist parallel constant mean curvature surfaces.

3.2. Gauss maps of integrable surfaces. In this subsection, we consider the Gauss maps of integrable surfaces defined in the previous section.

From [10, it is known that the complex Gauss map $N$ of a complex CMCimmersion $\Psi$ with null coordinates ( $N$ is also the complex Gauss map of the parallel complex CGC-immersion) satisfies the following equation:

$$
N_{z w}=\rho N \text {, }
$$


where $(z, w) \in \mathfrak{D}^{2} \subset \mathbb{C}^{2}$ and the function $\rho: \mathfrak{D}^{2} \rightarrow \mathbb{C}$ is defined by $\rho \cdot i \sigma_{3}=$ $\left[\alpha_{-1},\left[\alpha_{1}, i \sigma_{3}\right]\right]$ with $\alpha_{j}$ as defined in (3.1.2).

From Theorem 2.6. we note that the complex Gauss map $N$ is represented by $N=\frac{i}{2} \operatorname{Ad}(F) \sigma_{3}$, where $F$ is the complex extended framing of the complex CMCimmersion $\Psi$. Let $F^{(\mathfrak{c}, j)}$ (resp. $F^{(\mathfrak{s}, j)}$ ) be the extended framing of $\Phi^{(\mathfrak{c}, j)}$ for $j \in$ $\{1,2,3,4\}$ (resp. $\Phi^{(\mathfrak{s}, j)}$ for $j \in\{1,2,3\}$ ). Using (3.1.14), we can easily verify that $N^{(\mathfrak{c}, j)}$ and $N^{(\mathfrak{s}, j)}$ are maps into the following spaces:

$$
\left\{\begin{array}{l}
H^{2}=S U(1,1) / U(1) \text { for } j=1, \\
S^{1,1}=S L_{*}(2, \mathbb{R}) / K \text { for } j=2, \\
S^{2}=S U(2) / U(1) \text { for } j=3, \\
S L(2, \mathbb{C}) / U(1) \text { for } j=4
\end{array}\right.
$$

where $K=\left\{\operatorname{diag}\left[a, a^{-1}\right] \mid a \in \mathbb{R}^{*}\right\}$, which is isomorphic to $\mathbb{R}^{*}$, and $S L_{*}(2, \mathbb{R})=$ $\left\{g \in S L(2, \mathbb{C}) \mid g=\left(\begin{array}{ll}a & b \\ c & d\end{array}\right), a, d \in \mathbb{R}, b, c \in i \mathbb{R}\right\}$, which is isomorphic to $S L(2, \mathbb{R})$. It is known that the space $S L(2, \mathbb{C}) / U(1)$ is a 4 -symmetric space via the fourth order automorphism

$$
X \mapsto \operatorname{Ad}\left(\begin{array}{cc}
1 / \sqrt{i} & 0 \\
0 & \sqrt{i}
\end{array}\right)\left(\bar{X}^{t}\right)^{-1} \text { for } X \in S L(2, \mathbb{C}) .
$$

The choices of coordinates in (3.1.6), the functions in (3.1.7) and the relation $\rho i \sigma_{3}=$ $\left[\alpha_{-1}\left[\alpha_{1}, i \sigma_{3}\right]\right]$ imply

$$
\left(N^{(\mathfrak{c}, j)}\right)_{z \bar{z}}=\rho^{(\mathfrak{c}, j)} N^{(\mathfrak{c}, j)} \text { and }\left(N^{(\mathfrak{s}, j)}\right)_{x y}=\rho^{(\mathfrak{s}, j)} N^{(\mathfrak{s}, j)},
$$

where $\rho^{(\mathfrak{c}, j)}: \mathfrak{D} \subset \mathbb{C} \rightarrow \mathbb{R}$ and $\rho^{(\mathfrak{s}, j)}: \mathfrak{D} \subset \mathbb{R}^{2} \rightarrow \mathbb{R}$.

It is well known that the equations in (3.2.2) for $j \in\{1,2,3\}$ are equivalent to the harmonicities (resp. Lorentz harmonicities) of Gauss maps $N^{(\mathfrak{c}, j)}$ (resp. $\left.N^{(\mathfrak{s}, j)}\right)$ with respect to the second fundamental forms defined in the first equations of (3.1.15) (resp. third equations of (3.1.15)); see Theorem 13 in 20. We then have the following theorem:

Theorem 3.6. Let $\Phi^{(\mathfrak{c}, j)}$ for $j \in\{1,2,3,4\}$ and $\Phi^{(\mathfrak{s}, j)}$ for $j \in\{1,2,3\}$ be the integrable surfaces defined in Theorem 3.3, respectively. Moreover, let $N^{(\mathfrak{c}, j)}$ and $N^{(\mathfrak{s}, j)}$ be their Gauss maps, respectively. Then the Gauss maps $N^{(\mathfrak{c}, j)}$ and $N^{(\mathfrak{s}, j)}$ are characterized as follows:

$(C, S, 1)$ The Gauss map $N^{(\mathfrak{c}, 1)}$ (resp. $\left.N^{(\mathfrak{s}, 1)}\right)$ is an harmonic (resp. Lorentz harmonic) map into $H^{2}$.

$(C, S, 2)$ The Gauss map $N^{(\mathfrak{c}, 2)}$ (resp. $N^{(\mathfrak{s}, 2)}$ ) is an harmonic (resp. Lorentz harmonic) map into $S^{1,1}$.

$(C, S, 3)$ The Gauss map $N^{(\mathfrak{c}, 3)}$ (resp. $N^{(\mathfrak{s}, 3)}$ ) is an harmonic (resp. Lorentz harmonic) map into $S^{2}$

$(C, 4)$ The Gauss map $N^{(\mathfrak{c}, 4)}$ is an harmonic map into $S L(2, \mathbb{C}) / U(1)$.

Proof. Let us show the $(C, 4)$ case. We recall that a map from a Riemann surface into a $k$-symmetric space $N=G / K$ is harmonic (see, for example, [6, page 242]) if

$$
\begin{aligned}
& {\left[\alpha_{\mathfrak{m}}^{\prime} \wedge \alpha_{\mathfrak{m}}^{\prime \prime}\right]_{\mathfrak{m}}=0,} \\
& d \alpha_{\lambda}+\frac{1}{2}\left[\alpha_{\lambda} \wedge \alpha_{\lambda}\right]=0,
\end{aligned}
$$


TABLE 1. Integrable surfaces defined by the real forms of $\Lambda \mathfrak{s l}(2, \mathbb{C})_{\sigma}$

\begin{tabular}{|c|c|c|c|}
\hline Surfaces class & Gauss curvature & Gauss curvature & Parallel CMC \\
\hline Surfaces in $\mathbb{R}^{3}$ & $K^{(\mathfrak{s}, 3)}=-4|H|^{2}$ & $K^{(\mathfrak{c}, 3)}=4|H|^{2}$ & $H^{(\mathfrak{c}, 3)}=|H|$ \\
\hline Spacelike surfaces in $\mathbb{R}^{1,2}$ & $K^{(\mathfrak{s}, 1)}=4|H|^{2}$ & $K^{(\mathfrak{c}, 1)}=-4|H|^{2}$ & $H^{(\mathfrak{c}, 1)}=|H|$ \\
\hline Timelike surfaces in $\mathbb{R}^{1,2}$ & $K^{(\mathfrak{c}, 2)}=-4|H|^{2}$ & $K^{(\mathfrak{s}, 2)}=4|H|^{2}$ & $H^{(\mathfrak{s}, 2)}=|H|$ \\
\hline Surfaces in $H^{3}$ & & & $H^{(\mathfrak{c}, 4)}=\tanh (-q)$ \\
\hline
\end{tabular}

where $\alpha_{\lambda}=\lambda^{-1} \alpha_{\mathfrak{m}}^{\prime}+\alpha_{\mathfrak{k}}+\lambda \alpha_{\mathfrak{m}}^{\prime \prime}, \mathfrak{g}=\mathfrak{k} \oplus \mathfrak{m}$ is the reductive decomposition and ' (resp. ") denotes the (1,0)-part (resp. (0,1)-part). Since the map $N^{(\mathfrak{c}, 4)}$ has the lift $F^{(\mathfrak{c}, 4)}: \mathfrak{D} \rightarrow \Lambda S L(2, \mathbb{C})_{\sigma}^{(\mathfrak{c}, j)}$ which is defined from the complex extended framing $F$ with the conditions in (3.1.7), the Maurer-Cartan form $\alpha_{\lambda}=F^{(\mathfrak{c}, 4)-1} d F^{(\mathfrak{c}, 4)}$ has the form $\alpha_{\lambda}=\lambda^{-1} \alpha_{-1}+\alpha_{0}+\lambda \alpha_{1}$ and satisfies the Maurer-Cartan equation $d \alpha_{\lambda}+\frac{1}{2}\left[\alpha_{\lambda} \wedge \alpha_{\lambda}\right]=0$ (see (3.1.1) and Lemma 2.5). The conditions in (3.1.7) imply $\alpha_{0}=\alpha_{\mathfrak{k}}, \alpha_{-1}=\alpha_{\mathfrak{m}}^{\prime}$ and $\alpha_{1}=\alpha_{\mathfrak{m}}^{\prime \prime}$, where $\mathfrak{s l}(2, \mathbb{C})=\mathfrak{k} \oplus \mathfrak{m}$ is the reductive decomposition associated to $S L(2, \mathbb{C}) / U(1)$. Moreover, since $\alpha_{-1}$ and $\alpha_{1}$ have offdiagonal forms, it follows that $\left[\alpha_{\mathfrak{m}}^{\prime} \wedge \alpha_{\mathfrak{m}}^{\prime \prime}\right]_{\mathfrak{m}}=0$. Therefore, $N^{(\mathfrak{c}, 4)}$ is an harmonic map into the 4 -symmetric space $S L(2, \mathbb{C}) / U(1)$. For other cases, since the target spaces are symmetric spaces, the condition $\left[\alpha_{\mathfrak{m}}^{\prime} \wedge \alpha_{\mathfrak{m}}^{\prime \prime}\right]_{\mathfrak{m}}=0$ is vacuous. Thus the Maurer-Cartan equation $d \alpha_{\lambda}+\frac{1}{2}\left[\alpha_{\lambda} \wedge \alpha_{\lambda}\right]=0$ with $\alpha_{\lambda}=\lambda^{-1} \alpha_{-1}+\alpha_{0}+\lambda \alpha_{1}$ is equivalent to the map being harmonic or Lorentz harmonic. This completes the proof.

Remark 3.7. In fact, in the $(C, 4)$ case, the harmonic map $N^{(\mathfrak{c}, 4)}$ into the 4symmetric space $S L(2, \mathbb{C}) / U(1)$ is known as the so-called Legendre harmonic map [15]. We will discuss this topic in a separate publication [7].

\section{The GENERALIZED WEIERSTRAss TYPE REPRESENTATION FOR INTEGRABLE SURFACES}

The generalized Weierstrass type representation for complex CMC-immersions (or equivalently CGC-immersions as the parallel immersions) is the procedure of a construction of complex CMC-immersions from a pair of holomorphic potentials; see [10]. In the previous section, we classified all integrable surfaces according to the classification of real forms of $\Lambda \mathfrak{s l}(2, \mathbb{C})_{\sigma}$. In this section, we show how all integrable surfaces are obtained from the pairs of holomorphic potentials in the generalized Weierstrass type representation.

4.1. Integrable surfaces via the generalized Weierstrass type representation. The generalized Weierstrass type representation for complex CMC-immersions (or, equivalently, CGC-immersions as the parallel immersions) is divided into the following 4 steps; see also [10] for more details:

Step 1: Let $\check{\eta}=(\eta(z, \lambda), \tau(w, \lambda))$ be a pair of holomorphic potentials of the following forms:

$$
\check{\eta}=(\eta(z, \lambda), \quad \tau(w, \lambda))=\left(\sum_{k=-1}^{\infty} \eta_{k}(z) \lambda^{k}, \quad \sum_{m=-\infty}^{1} \tau_{m}(w) \lambda^{m}\right),
$$


where $(z, w) \in \mathfrak{D}^{2}$ and where $\mathfrak{D}^{2}$ is some holomorphically convex domain in $\mathbb{C}^{2}, \lambda \in \mathbb{C}^{*},|\lambda|=r(0<r<1)$, and $\eta_{k}$ and $\tau_{m}$ are $\mathfrak{s l}(2, \mathbb{C})$-valued holomorphic differential 1-forms. Moreover, $\eta_{k}(z)$ and $\tau_{k}(w)$ are diagonal (resp. off-diagonal) matrices if $k$ is even (resp. odd). We also assume that the upper right entry of $\eta_{-1}(z)$ and the lower left entry $\tau_{1}(w)$ do not vanish for all $(z, w) \in \mathfrak{D}^{2}$.

Step 2: Let $C$ and $L$ denote the solutions to the following linear ordinary differential equations

$$
d C=C \eta \text { and } d L=L \tau \text { with } C\left(z_{*}, \lambda\right)=L\left(w_{*}, \lambda\right)=\mathrm{id},
$$

where $\left(z_{*}, w_{*}\right) \in \mathfrak{D}^{2}$ is a fixed base point.

Step 3: We factor the pair of matrices $(C, L)$ via the generalized Iwasawa decomposition of Theorem A.2 as follows:

$$
(C, L)=(F, F)(\mathrm{id}, W)\left(V_{+}, V_{-}\right),
$$

where $V_{ \pm} \in \Lambda^{ \pm} S L(2, \mathbb{C})_{\sigma}$.

Theorem 4.1 ([10). Let $F$ be a $\Lambda S L(2, \mathbb{C})_{\sigma}$-loop defined by the generalized Iwasawa decomposition in (4.1.3). Then there exists a $\lambda$-independent diagonal matrix $l(z, w) \in S L(2, \mathbb{C})$ such that $F \cdot l$ is a complex extended framing of some complex $\mathrm{CMC}$-immersion (or, equivalently, the complex $\mathrm{CGC}$-immersion as the parallel immersion).

Step 4: The Sym formula defined in 2.2.1 via $F(z, w, \lambda) l(z, w)$ represents a complex CMC-immersion and a complex CGC-immersion in $\mathfrak{s l}(2, \mathbb{C}) \cong \mathbb{C}^{3}$.

Let $\mathfrak{c}_{j}$ for $j \in\{1,2,3,4\}$ and $\mathfrak{s}_{j}$ for $j \in\{1,2,3\}$ be the involutions defined in (3.1.3), respectively. Then we define the following pairs of involutions on $\check{\eta}=$ $(\eta, \tau) \in \Omega\left(\Lambda \mathfrak{s l}(2, \mathbb{C})_{\sigma}\right) \times \Omega\left(\Lambda \mathfrak{s l}(2, \mathbb{C})_{\sigma}\right):$

$$
\mathfrak{r}_{j}:(\eta, \tau) \longmapsto\left(\mathfrak{c}_{j} \tau, \mathfrak{c}_{j} \eta\right) \text { and } \mathfrak{d}_{j}:(\eta, \tau) \longmapsto\left(\mathfrak{s}_{j} \eta, \mathfrak{s}_{j} \tau\right) \text {. }
$$

We now prove the following theorem.

Theorem 4.2. Let $\check{\eta}=(\eta(z, \lambda), \tau(w, \lambda))$ be a pair of holomorphic potentials defined as in (4.1.1), and let $\mathfrak{r}_{j}$ for $j \in\{1,2,3,4\}$ and $\mathfrak{d}_{j}$ for $j \in\{1,2,3\}$ be the pairs of involutions defined in (4.1.4), respectively. Then the following statements hold:

$(C, 1)$ If $\mathfrak{r}_{1}(\check{\eta})=\check{\eta}$, then the resulting immersions given by the generalized Weierstrass type representation are spacelike constant negative Gaussian curvature surfaces in $\mathbb{R}^{1,2}$.

$(C, 2)$ If $\mathfrak{r}_{2}(\check{\eta})=\check{\eta}$, then the resulting immersions given by the generalized Weierstrass type representation are timelike constant negative Gaussian curvature surfaces in $\mathbb{R}^{1,2}$.

$(C, 3)$ If $\mathfrak{r}_{3}(\check{\eta})=\check{\eta}$, then the resulting immersions given by the generalized Weierstrass type representation are constant positive Gaussian curvature surfaces in $\mathbb{R}^{3}$.

$(C, 4)$ If $\mathfrak{r}_{4}(\check{\eta})=\check{\eta}$, then the resulting immersions given by the generalized Weierstrass type representation are constant mean curvature surfaces with mean curvature $\left|H^{(\mathfrak{c}, 4)}\right|<1$ in $H^{3}$.

$(S, 1)$ If $\mathfrak{d}_{1}(\check{\eta})=\check{\eta}$, then the resulting immersions given by the generalized Weierstrass type representation are spacelike constant positive Gaussian curvature surfaces in $\mathbb{R}^{1,2}$. 
$(S, 2)$ If $\mathfrak{d}_{2}(\check{\eta})=\check{\eta}$, then the resulting immersions given by the generalized Weierstrass type representation are timelike constant positive Gaussian curvature surfaces in $\mathbb{R}^{1,2}$.

$(S, 3)$ If $\mathfrak{d}_{3}(\check{\eta})=\check{\eta}$, then the resulting immersions given by the generalized Weierstrass type representation are constant negative Gaussian curvature surfaces in $\mathbb{R}^{3}$.

Proof. Since the pairs of holomorphic potentials are invariant under the involutions $\mathfrak{r}_{j}$ or $\mathfrak{d}_{j}$, the coordinates $(z, w) \in \mathfrak{D}^{2}$ satisfy the following relations:

$$
\left\{\begin{array}{l}
w=\bar{z} \text { if } \mathfrak{r}_{j}(\check{\eta})=\check{\eta} \\
z=\bar{z} \text { and } w=\bar{w} \text { if } \mathfrak{d}_{j}(\check{\eta})=\check{\eta} .
\end{array}\right.
$$

Let $(C, L)$ be the pair of solutions of the differential equations in (4.1.2) with the initial conditions $C\left(z_{*}\right)=L\left(w_{*}\right)=\mathrm{id}$, where $\left(z_{*}, w_{*}\right) \in \mathfrak{D}^{2}$ satisfies one of the conditions in (4.1.5). Let $\mathcal{R}_{j}$ for $j \in\{1,2,3,4\}$ (resp. $\mathcal{D}_{j}$ for $j \in\{1,2,3\}$ ) be the following pair of involutions on $\Lambda S L(2, \mathbb{C})_{\sigma} \times \Lambda S L(2, \mathbb{C})_{\sigma}$ :

$$
\mathcal{R}_{j}(C, L):=\left(\mathcal{C}_{j}(L), \mathcal{C}_{j}(C)\right) \text { and } \mathcal{D}_{j}(C, L):=\left(\mathcal{S}_{j}(C), \mathcal{S}_{j}(L)\right),
$$

where $\mathcal{C}_{j}\left(\right.$ resp. $\left.\mathcal{S}_{j}\right)$ are the involutions on $\Lambda S L(2, \mathbb{C})_{\sigma}$ corresponding to the involutions $\mathfrak{c}_{j}$ (resp. $\mathfrak{s}_{j}$ ) as in Theorem 2.10 (resp. Theorem 2.11), e.g.,

$$
\mathcal{C}_{1}: C(\lambda) \rightarrow \overline{C(-1 / \bar{\lambda}}^{t-1} \text { and } \mathcal{S}_{1}: C(\lambda) \rightarrow \overline{C(-\bar{\lambda})}^{t-1} \text { for } C(\lambda) \in \Lambda S L(2, \mathbb{C})_{\sigma}
$$

Noting the conditions in (4.1.5), the involutions $\mathfrak{r}_{j}$ for $j \in\{1,2,3,4\}$ and $\mathfrak{d}_{j}$ for $j \in\{1,2,3\}$ in (4.1.4) define symmetries on the pair of solutions $(C, L)$ as follows: (4.1.7)

$$
\left\{\begin{array}{l}
\mathcal{R}_{j}(C(z, \lambda), L(\bar{z}, \lambda))=(C(z, \lambda), L(\bar{z}, \lambda)) \text { if } \mathfrak{r}_{j}(\check{\eta})=\check{\eta} \text { for } j \in\{1,2,3,4\}, \\
\mathcal{D}_{j}(C(x, \lambda), L(y, \lambda))=(C(x, \lambda), L(y, \lambda)) \text { if } \mathfrak{d}_{j}(\check{\eta})=\check{\eta} \text { for } j \in\{1,2,3\},
\end{array}\right.
$$

where $x=z=\bar{z} \in \mathbb{R}$ and $y=w=\bar{w} \in \mathbb{R}$. Applying the generalized Iwasawa decomposition of Theorem A.2 for $(C, L) \in \Lambda S L(2, \mathbb{C})_{\sigma} \times \Lambda S L(2, \mathbb{C})_{\sigma}$, we have

$$
(C, L)=(F, F)(\mathrm{id}, W)\left(V_{+}, V_{-}\right),
$$

where $V_{ \pm} \in \Lambda^{ \pm} S L(2, \mathbb{C})_{\sigma}$. If $(z, w) \in \mathfrak{D}^{2}$ is sufficiently close to $\left(z_{*}, w_{*}\right) \in \mathfrak{D}^{2}$, then the middle term $W$ of the generalized Iwasawa decomposition is the identity.

Since the left component $F$ of the generalized Iwasawa decomposition in (4.1.8) can be rephrased as $F=C V_{+}^{-1}=L V_{-}^{-1}$, we have

$$
C^{-1} L=V_{+}^{-1} V_{-} \text {. }
$$

From the symmetries on $(C, L)$ in (4.1.7), $V_{-}$and $V_{+}$have the following relations:

$$
\left\{\begin{array}{l}
\mathcal{C}_{j}\left(V_{ \pm}(z, \bar{z}, \lambda)\right)=k^{(\mathfrak{c}, j)}(z, \bar{z})^{-1} V_{\mp}(z, \bar{z}, \lambda) \text { if } \mathfrak{r}_{j}(\check{\eta})=\check{\eta} \text { for } j \in\{1,2,3,4\}, \\
\mathcal{S}_{j}\left(V_{ \pm}(x, y, \lambda)\right)=k^{(\mathfrak{s}, j)}(x, y)^{-1} V_{ \pm}(x, y, \lambda) \text { if } \mathfrak{d}_{j}(\check{\eta})=\check{\eta} \text { for } j \in\{1,2,3\},
\end{array}\right.
$$

where $k^{(\mathfrak{c}, j)}(z, \bar{z})$ and $k^{(\mathfrak{s}, j)}(x, y)$ are $\lambda$-independent diagonal matrices satisfying the symmetries $\mathcal{C}_{j}\left(k^{(\mathfrak{c}, j)}(z, \bar{z})\right)=k^{(\mathfrak{c}, j)}(z, \bar{z})^{-1}$ and $\mathcal{S}_{j}\left(k^{(\mathfrak{s}, j)}(x, y)\right)=k^{(\mathfrak{s}, j)}(x, y)^{-1}$, respectively. From the discussion above, $F$ has the symmetry as follows:

$$
\left\{\begin{array}{l}
\mathcal{C}_{j}(F(z, \bar{z}, \lambda))=F(z, \bar{z}, \lambda) k^{(\mathfrak{c}, j)}(z, \bar{z}) \text { if } \mathfrak{r}_{j}(\check{\eta})=\check{\eta} \text { for } j \in\{1,2,3,4\} \\
\mathcal{S}_{j}(F(x, y, \lambda))=F(x, y, \lambda) k^{(\mathfrak{s}, j)}(x, y) \text { if } \mathfrak{d}_{j}(\check{\eta})=\check{\eta} \text { for } j \in\{1,2,3\}
\end{array}\right.
$$


Let $F^{(\mathfrak{c}, j)}$ (resp. $F^{(\mathfrak{s}, j)}$ ) denote the left components $F$ of the generalized Iwasawa decomposition in (4.1.8) which have the symmetries in (4.1.9) by $\mathcal{C}_{j}$ (resp. $\left.\mathcal{S}_{j}\right)$. Let $\tilde{k}^{(\mathfrak{c}, j)}(z, \bar{z})$ and $\tilde{k}^{(\mathfrak{s}, j)}(x, y)$ be the $\lambda$-independent diagonal matrices such that $\tilde{k}^{(\mathfrak{c}, j)}(z, \bar{z})^{2}=k^{(\mathfrak{c}, j)}(z, \bar{z})$ and $\tilde{k}^{(\mathfrak{s}, j)}(x, y)^{2}=k^{(\mathfrak{s}, j)}(x, y)$, respectively. Setting $\tilde{F}^{(\mathfrak{c}, j)}(z, \bar{z}, \lambda)=F^{(\mathfrak{c}, j)}(z, \bar{z}, \lambda) \tilde{k}^{(\mathfrak{c}, j)}(z, \bar{z})$ and $\tilde{F}^{(\mathfrak{s}, j)}(x, y, \lambda)=F^{(\mathfrak{s}, j)}(x, y, \lambda) \tilde{k}^{(\mathfrak{s}, j)}(x, y)$, we have

$$
\left\{\begin{array}{l}
\mathcal{C}_{j}\left(\tilde{F}^{(\mathfrak{c}, j)}(z, \bar{z}, \lambda)\right)=\tilde{F}^{(\mathfrak{c}, j)}(z, \bar{z}, \lambda) \text { if } \quad \mathfrak{r}_{j}(\check{\eta})=\check{\eta} \text { for } j \in\{1,2,3,4\}, \\
\mathcal{S}_{j}\left(\tilde{F}^{(\mathfrak{s}, j)}(x, y, \lambda)\right)=\tilde{F}^{(\mathfrak{s}, j)}(x, y, \lambda) \text { if } \mathfrak{d}_{j}(\check{\eta})=\check{\eta} \text { for } j \in\{1,2,3\} .
\end{array}\right.
$$

Moreover, a straightforward calculation shows that $\alpha^{(\mathfrak{c}, j)}:=\tilde{F}^{(\mathfrak{c}, j)-1} d \tilde{F}^{(\mathfrak{c}, j)}$ and $\alpha^{(\mathfrak{s}, j)}:=\tilde{F}^{(\mathfrak{s}, j)-1} d \tilde{F}^{(\mathfrak{s}, j)}$ have the forms in (3.1.1) with the properties in (3.1.7), i.e., $\tilde{F}^{(\mathfrak{c}, j)} \in \Lambda S L(2, \mathbb{C})_{\sigma}^{(\mathfrak{c}, j)}$ and $\tilde{F}^{(\mathfrak{s}, j)} \in \Lambda S L(2, \mathbb{C})_{\sigma}^{(\mathfrak{s}, j)}$ are the extended framings. From the argument in Theorem 3.3, the Sym formulas $\Phi^{(\mathfrak{c}, j)}$ for $j \in\{1,2,3\}$ in (3.1.9) via $\tilde{F}^{(\mathfrak{c}, j)}, \Phi^{(\mathfrak{c}, 4)}$ in (3.1.10) via $\tilde{F}^{(\mathfrak{c}, 4)}$ and $\Phi^{(\mathfrak{s}, j)}$ for $j \in\{1,2,3\}$ in (3.1.11) via $\tilde{F}^{(\mathfrak{s}, j)}$ define immersions which have the properties as desired. This completes the proof.

Remark 4.3. From the forms of pairs of involutions $\mathfrak{r}_{j}$ for $j \in\{1,2,3,4\}$ defined in (4.1.4), the pairs of holomorphic potentials $\check{\eta}$ for $(C, j)$ cases in Theorem 4.2 are generated by a single potential, i.e., $\check{\eta}=(\eta, \tau)=\left(\eta, \mathfrak{c}_{j}(\eta)\right)$, where $\mathfrak{c}_{j}$ for $j \in$ $\{1,2,3,4\}$ are involutions defined in (3.1.3).

Remark 4.4. In the proof of Theorem 4.2, we assume our domain $\mathfrak{D}^{2} \subset \mathbb{C}^{2}$ is sufficiently small around the initial point $\left(z_{*}, w_{*}\right) \in \mathfrak{D}^{2}$ so that the middle term $w_{n}$ of the generalized Iwasawa decomposition of Theorem A.2 is in the identity component. In general, if we consider the larger domain $\widetilde{\mathfrak{D}}^{2}$ such that $\mathfrak{D}^{2} \subset \widetilde{\mathfrak{D}}^{2}$, then the middle terms $w_{n}$ have many components. Therefore the extended framing $F$ could have singularities on $\widetilde{\mathfrak{D}}^{2}$.

\section{ApPEndix A. BASIC NOTATION AND RESUlts For AFFine KaC-Moody Lie Algebras and Lie Groups}

A.1. Kac-Moody Lie algebras. We first recall the definition of the generalized Cartan matrix, which is an $n \times n$ matrix $A=\left(a_{i j}\right)$ satisfying the following conditions:

(1) For $i \in\{1, \ldots, n\}, a_{i i}=2$.

(2) For $i \neq j, a_{i j} \leq 0$.

(3) For $i \neq j$, if $a_{i j}=0$, then $a_{j i}=0$.

The generalized Cartan matrix $A$ is called symmetrizable if $A$ can be decomposed as $A=D S$, where $D$ is a positive definite diagonal matrix and $S$ is a symmetric matrix. The generalized Cartan matrix $A=\left(a_{i j}\right)$ is called decomposable if there exists $\sigma \in S_{n}$ such that $\left(a_{\sigma(i) \sigma(j)}\right)=\left(\begin{array}{cc}B & 0 \\ 0 & C\end{array}\right)$, where $B$ and $C$ are square matrices. If $A$ is not decomposable, then $A$ is called indecomposable. The symmetrizable and indecomposable generalized Cartan matrices are classified into finite, affine and indefinite if $S\left(=D^{-1} A\right)$ is positive definite, positive semidefinite and indefinite, respectively; see [16, Chapter 4].

Let $A=\left(a_{i j}\right)$ be the generalized Cartan matrix of rank $r$, and let $\mathfrak{h}$ be a vector space over the complex field $\mathbb{C}$ such that $\operatorname{dimh}=n+\operatorname{corank}(A)$. Moreover, let $\Pi=\left\{\alpha_{1}, \ldots, \alpha_{n}\right\}$ and $\check{\Pi}=\left\{\check{\alpha}_{1}, \ldots, \check{\alpha}_{n}\right\}$ be linearly independent in $\mathfrak{h}^{*}$ and $\mathfrak{h}$, 
respectively, such that $\alpha_{j}\left(\check{\alpha}_{i}\right)=a_{i j}$. It follows that if $A$ is nonsingular, then $\check{\Pi}$ (resp. $\Pi$ ) is a basis of $\mathfrak{h}\left(\right.$ resp. $\left.\mathfrak{h}^{*}\right)$. It is well known that the $\alpha_{i}$ (resp. $\check{\alpha}_{i}$ ) for $i \in\{1,2, \ldots, n\}$ are known as the roots (resp. the coroots).

We now define the complex Kac-Moody algebra $\mathfrak{g}=\mathfrak{g}(A)$ associated to $A$ : it is generated by $\left\{\mathfrak{h}, e_{i}, f_{i} ; i=1,2, \ldots, n\right\}$ with the following relations:

$$
\begin{array}{lll}
{[\mathfrak{h}, \mathfrak{h}]=0,} & {\left[e_{i}, f_{j}\right]=\delta_{i j} \check{\alpha}_{i}} & (i, j=1,2, \ldots, n), \\
{\left[h, e_{i}\right]=\alpha_{i}(h) e_{i},} & {\left[h, f_{i}\right]=-\alpha_{i}(h) f_{i}} & (h \in \mathfrak{h}), \\
\left(\operatorname{ad} e_{i}\right)^{1-a_{i j}}\left(e_{j}\right)=0, & \left(\operatorname{ad} f_{i}\right)^{1-a_{i j}}\left(f_{j}\right)=0 & (i \neq j) .
\end{array}
$$

The Kac-Moody algebra $\mathfrak{g}=\mathfrak{g}(A)$ is said to be of finite, affine or indefinite type if the corresponding generalized Cartan matrix $A$ is as well. We note that $\mathfrak{h}$ is known as the Cartan subalgebra of the Kac-Moody algebra $\mathfrak{g}(A)$.

For a finite dimensional Lie algebra and its Cartan matrix, the extended Cartan matrix can be defined by adding a zero'th row and column to the Cartan matrix, corresponding to adding a new simple root $\alpha_{0}:=-\theta$, where $\theta$ is the maximal root for $\mathfrak{g}$ with respect to $\Pi=\left\{\alpha_{1}, \ldots, \alpha_{n}\right\}$. It is known that the extended Cartan matrix is an example of the generalized Cartan matrix of affine type [16.

A.2. Loop algebras and loop groups. In this subsection, we introduce a loop group and a loop algebra, and we give a characterization of the loop algebras via affine Kac-Moody Lie algebras. Let $C_{r}:=\{\lambda \in \mathbb{C}|| \lambda \mid=r\}$ be the circle of radius $r$ with $r \in(0,1]$, and let $D_{r}:=\{\lambda \in \mathbb{C}|| \lambda \mid<r\}$ be the open disk of radius $r$. Let $G$ be a Lie group and let $\mathfrak{g}$ be its Lie algebra. For any $r \in(0,1] \subset \mathbb{R}$, we consider the twisted loop algebra and loop group:

$$
\begin{aligned}
& \Lambda_{r} \mathfrak{g}_{\sigma}=\left\{\alpha: C_{r} \rightarrow \mathfrak{g} \mid \alpha \text { is continuous and } \alpha(-\lambda)=\sigma_{3} \alpha(\lambda) \sigma_{3}\right\}, \\
& \Lambda_{r} G_{\sigma}=\left\{g: C_{r} \rightarrow G \mid g \text { is continuous and } g(-\lambda)=\sigma_{3} g(\lambda) \sigma_{3}\right\},
\end{aligned}
$$

where $\sigma_{3}$ is defined in (2.0.2).

Let $\mathcal{A}$ be the "Wiener algebra"

$$
\mathcal{A}=\left\{f(\lambda)=\sum_{n \in \mathbb{Z}} f_{n} \lambda^{n}: C_{r} \rightarrow \mathbb{C} ; \sum_{n \in \mathbb{Z}}\left|f_{n}\right|<\infty\right\} .
$$

The Wiener algebra is a Banach algebra relative to the norm $\|f\|=\sum\left|f_{n}\right|$, and $\mathcal{A}$ consists of continuous functions. Thus the loop groups and loop algebras with coefficients in $\mathcal{A}$ are Banach Lie groups and Banach Lie algebras.

From [16, we quote the following realization of the affine Kac-Moody Lie algebras via loop algebras:

Theorem A.1 ([16]). Let $\mathfrak{g}$ be a complex finite dimensional simple Lie algebra, let $A$ be its extended Cartan matrix, and let $\Lambda \mathfrak{g}$ be its loop algebra. Moreover, let $\tilde{\Lambda} \mathfrak{g}:=\Lambda \mathfrak{g} \oplus \mathbb{C} k \oplus \mathbb{C} c$ be the twice central extension of the loop algebra $\Lambda \mathfrak{g}$. Then $\tilde{\Lambda} \mathfrak{g}$ is the affine Kac-Moody Lie algebra associated to the generalized Cartan matrix A of affine type.

A.3. Double loop groups and the generalized Iwasawa decompositions. In this subsection, we give the basic notation and results for double loop groups; see 12 for more details. Let $D_{r}:=\{\lambda \in \mathbb{C}|| \lambda \mid<r\}$ be an open disk and denote the closure of $D_{r}$ by $\overline{D_{r}}:=\{\lambda \in \mathbb{C}|| \lambda \mid \leq r\}$. Also, let $A_{r}=\{\lambda \in \mathbb{C}|r<| \lambda \mid<1 / r\}$ be an open annulus containing $S^{1}$, and denote the closure of $A_{r}$ by $\overline{A_{r}}$. Furthermore, let $E_{r}=\{\lambda \in \mathbb{C}|r<| \lambda \mid\} \cup\{\infty\}$ be an exterior of the circle $C_{r}$. 
We recall the definitions of the twisted plus $r$-loop group and the minus $r$-loop group of $\Lambda S L(2, \mathbb{C})_{\sigma}$ as follows:

$$
\begin{aligned}
\Lambda_{r, B}^{+} S L(2, \mathbb{C})_{\sigma} & :=\left\{W_{+} \in \Lambda_{r} S L(2, \mathbb{C})_{\sigma} \mid \begin{array}{r}
W_{+}(\lambda) \text { extends holomorphically } \\
\text { to } D_{r} \text { and } W_{+}(0) \in \boldsymbol{B}
\end{array}\right\}, \\
\Lambda_{r, B}^{-} S L(2, \mathbb{C})_{\sigma} & :=\left\{W_{-} \in \Lambda_{r} S L(2, \mathbb{C})_{\sigma} \mid \begin{array}{r}
W_{-}(\lambda) \text { extends holomorphically } \\
\text { to } E_{r} \text { and } W_{-}(\infty) \in \boldsymbol{B}
\end{array}\right\},
\end{aligned}
$$

where $\boldsymbol{B}$ is a subgroup of $S L(2, \mathbb{C})$. If $\boldsymbol{B}=$ id we write the subscript $*$ instead of $\boldsymbol{B}$, if $\boldsymbol{B}=S L(2, \mathbb{C})$ we abbreviate $\Lambda_{r, B}^{+} S L(2, \mathbb{C})_{\sigma}$ and $\Lambda_{r, B}^{-} S L(2, \mathbb{C})_{\sigma}$ by $\Lambda_{r}^{+} S L(2, \mathbb{C})_{\sigma}$ and $\Lambda_{r}^{-} S L(2, \mathbb{C})_{\sigma}$, respectively. From now on we will use the subscript $\boldsymbol{B}$ as above only if $\boldsymbol{B} \cap S U(2)=$ id holds. When $r=1$, we always omit the 1 .

We set the product of two loop groups:

$$
\mathcal{H}=\Lambda_{r} S L(2, \mathbb{C})_{\sigma} \times \Lambda_{R} S L(2, \mathbb{C})_{\sigma}
$$

where $0<r<R$. Moreover, we set the subgroups of $\mathcal{H}$ as follows:

$$
\begin{aligned}
& \mathcal{H}_{+}=\Lambda_{r}^{+} S L(2, \mathbb{C})_{\sigma} \times \Lambda_{R}^{-} S L(2, \mathbb{C})_{\sigma}, \\
& \mathcal{H}_{-}=\left\{\left(g_{1}, g_{2}\right) \in \mathcal{H} \mid \begin{array}{r}
g_{1} \text { and } g_{2} \text { extend holomorphically } \\
\text { to } A_{r} \text { and }\left.g_{1}\right|_{A_{r}}=\left.g_{2}\right|_{A_{r}}
\end{array}\right\} .
\end{aligned}
$$

We then quote Theorem 2.6 in 12 .

Theorem A.2. $\mathcal{H}_{-} \times \mathcal{H}_{+} \rightarrow \mathcal{H}_{-} \mathcal{H}_{+}$is an analytic diffeomorphism. The image is open and dense in $\mathcal{H}$. More precisely,

$$
\mathcal{H}=\bigcup_{n=0}^{\infty} \mathcal{H}_{-} w_{n} \mathcal{H}_{+}
$$

where $w_{n}=\left(\mathrm{id},\left(\begin{array}{cc}\lambda^{n} & 0 \\ 0 & \lambda^{-n}\end{array}\right)\right)$ if $n=2 k$ and $\left(\mathrm{id},\left(\begin{array}{cc}0 & \lambda^{n} \\ -\lambda^{-n} & 0\end{array}\right)\right)$ if $n=2 k+1$.

The proof of the theorem above is almost verbatim the proof given in the basic decomposition paper [5]; see also [9].

\section{REFERENCES}

[1] Algèbres de Kac-Moody affines, volume 11 of Institut Élie Cartan. Université de Nancy Institut Élie Cartan, Nancy, 1989. Automorphismes et formes réeles. [Automorphisms and real forms], Articles by Jean Bausch and Guy Rousseau. MR.1021052 (91a:17037)

[2] M. Babich and A. Bobenko. Willmore tori with umbilic lines and minimal surfaces in hyperbolic space. Duke Math. J., 72(1):151-185, 1993. MR1242883 (94j:53070)

[3] V. Back-Valente, N. Bardy-Panse, H. Ben Messaoud, and G. Rousseau. Formes presquedéployées des algèbres de Kac-Moody: classification et racines relatives. J. Algebra, 171(1):4396, 1995. MR:1314093 (96d:17022)

[4] H. Ben Messaoud and G. Rousseau. Classification des formes réelles presque compactes des algèbres de Kac-Moody affines. J. Algebra, 267(2):443-513, 2003. MR2003338(2004h:17028)

[5] M. J. Bergvelt and M. A. Guest. Actions of loop groups on harmonic maps. Trans. Amer. Math. Soc., 326(2):861-886, 1991. MR 1062870(91k:58022)

[6] F. E. Burstall and F. Pedit. Harmonic maps via Adler-Kostant-Symes theory. In Harmonic maps and integrable systems, Aspects Math., E23, pages 221-272. Vieweg, Braunschweig, 1994. MR1264189

[7] J. Dorfmeister, J. Inoguchi, and S.-P. Kobayashi. Constant mean curvature surfaces in hyperbolic 3-space via loop groups. Preprint, 2009. 
[8] J. Dorfmeister, J. Inoguchi, and M. Toda. Weierstrass-type representation of timelike surfaces with constant mean curvature. In Differential geometry and integrable systems (Tokyo, 2000), volume 308 of Contemp. Math., pages 77-99. Amer. Math. Soc., Providence, RI, 2002. MR.1955630(2003m:53010)

[9] J. Dorfmeister and S.-P. Kobayashi. Coarse classification of constant mean curvature cylinders. Trans. Amer. Math. Soc., 359(6):2483-2500 (electronic), 2007. MR2286041 (2008e:53011)

[10] J. Dorfmeister, S.-P. Kobayashi, and F. Pedit. Complex surfaces of constant mean curvature fibered by minimal surfaces. Hokkaido Math. J., 39(1):1-55, 2010. MR2649325

[11] J. Dorfmeister, F. Pedit, and H. Wu. Weierstrass type representation of harmonic maps into symmetric spaces. Comm. Anal. Geom., 6(4):633-668, 1998. MR1664887(2000d:53099)

[12] J. Dorfmeister and $\mathrm{H}$. Wu. Constant mean curvature surfaces and loop groups. J. Reine Angew. Math., 440:43-76, 1993. MR:1225957(94j:53005)

[13] J. Inoguchi. Timelike surfaces of constant mean curvature in Minkowski 3-space. Tokyo J. Math., 21(1):141-152, 1998. MR1630159(99e:53098)

[14] J. Inoguchi. Surfaces in Minkowski 3-space and harmonic maps. In Harmonic morphisms, harmonic maps, and related topics (Brest, 1997), volume 413 of Chapman \& Hall/CRC Res. Notes Math., pages 249-270. Chapman \& Hall/CRC, Boca Raton, FL, 2000.

[15] T. Ishihara. The harmonic Gauss maps in a generalized sense. J. London Math. Soc. (2), 26(1):104-112, 1982. MR667248 (84c:53053)

[16] V. G. Kac. Infinite-dimensional Lie algebras. Cambridge University Press, Cambridge, third edition, 1990. MR1104219(92k:17038)

[17] Z. Kobayashi. Automorphisms of finite order of the affine Lie algebra $A_{l}^{(1)}$. Tsukuba J. Math., 10(2):269-283, 1986. MR868654(87m:17034)

[18] C. LeBrun. Spaces of complex null geodesics in complex-Riemannian geometry. Trans. Amer. Math. Soc., 278(1):209-231, 1983. MR697071 (84e:32023)

[19] M. Melko and I. Sterling. Application of soliton theory to the construction of pseudospherical surfaces in $\mathbf{R}^{3}$. Ann. Global Anal. Geom., 11(1):65-107, 1993. MR1201412 (94a:53018)

[20] T. K. Milnor. Harmonic maps and classical surface theory in Minkowski 3-space. Trans. Amer. Math. Soc., 280(1):161-185, 1983. MR712254(85e:58037)

[21] B. O'Neill. Semi-Riemannian geometry, volume 103 of Pure and Applied Mathematics. Academic Press Inc. [Harcourt Brace Jovanovich Publishers], New York, 1983. MR0719023 (85f:53002)

[22] T. Weinstein. An introduction to Lorentz surfaces, volume 22 of de Gruyter Expositions in Mathematics. Walter de Gruyter \& Co., Berlin, 1996. MR1405166 (98a:53104)

Graduate School of Science and Technology, Hirosaki University, Bunkyocho 3 AoMORI 036-8561 JAPAN

E-mail address: shimpei@cc.hirosaki-u.ac.jp 